\title{
Photostriction and elasto-optic response in multiferroics and ferroelectrics from first principles
}

\author{
Yurong Yang ${ }^{1}$, Charles Paillard ${ }^{1}$, Bin $\mathrm{Xu}^{1}$, and L. Bellaiche ${ }^{1}$ \\ ${ }^{1}$ Department of Physics and nstitute for Nanoscience and \\ Engineering, University of Arkansas, Fayetteville, Arkansas 72701, \\ USA
}

November 11, 2017

\begin{abstract}
The present work reviews a series of recent first-principles studies devoted to the description of the interaction of light and strain in ferroelectric and multiferroic materials. Specifically, the modelling schemes used in these works to describe the so-called photostriction and elasto-optic effects are presented, in addition to the results and analysis provided by these $a b$ initio calculations. In particular, the large importance of the piezoelectric effect in the polar direction in the photostriction of ferroelectric materials is stressed out. Similarly, the occurrence of low-symmetry phases in lead titanate thin films under tensile strain is demonstrated to result in large elasto-optic constants. In addition, first-principle calculations allow to gain microscopic knowledge of subtle effects, for instance in the case of photostriction, where the deformation potential effect in directions perpendicular to the polar axis is shown to be almost as significant as the piezoelectric effect. As a result, the numerical methods presented here could propel the design of efficient opto-mechanical devices.
\end{abstract}

\section{Introduction}

\subsection{Ferroelectric and Multiferroic Materials}

Perovskite ferroelectrics possess a spontaneous electrical polarization and form an important class of materials [1]. Such compounds exhibit properties of technological relevance, since they can, e.g., convert various forms of energies. For instance, some ferroelectric (FE) systems can efficiently convert mechanical to electrical energies and vice-versa, because of their large piezoelectric coefficients, which is at the heart of piezoelectric transducers and actuators. Ferroelectric 
materials are also of importance for other applications, such as non-volatile memories, dielectrics for microelectronics and wireless communication, and pyroelectric arrays

Perovskite multiferroics (MFEs) constitute another intensively studied type of materials because of the inherent coexistence between ferroelectricity and magnetism. The primary initial interest of MFE materials resides in the promise of designing new devices exploiting the magnetoelectric coupling between magnetic and electrical properties [2]. As a matter of fact, controlling magnetic properties by an electric field would be of large technological interest.

There are properties and phenomena of FEs and MFEs that have been much less studied than, e.g., piezoelectricity and magnetoelectricity, despite the numerous studies devoted to these two types of materials. Such properties and phenomena are related to the interaction of light and matter, and hold promise for exciting discoveries - such as the use of ferroelectrics as novel and efficient photovoltaic or photoconductive materials $[3,4,5]$.

In this Review article, we will concentrate on recent computational, ab-initio efforts devoted to two specific light-matter interactions, that are photostriction and elasto-optic effects.

\subsection{Photostriction}

Most ferroelectric materials are semiconductors or insulators, and thus have a finite bandgap. As such, when light made of photons with energy higher than the bandgap hits a ferroelectric material, it is partially absorbed. Its absorption can convert the energy carried by light into:

(i) Electrical energy, i.e., the photovoltaic effect, whose origin may stem from various contributions [6, 7]: the so-called Bulk Photovoltaic Effect (BPVE) generated by the lack of inversion symmetry inherent to all ferroelectric materials $[8,9,10,11]$, the ferroelectric-metal Schottky interface, the presence of internal electric fields caused by defects, etc.

(ii) Chemical energy, i.e., the photocatalysis effect [12].

(iii) Mechanical energy, i.e., the photostriction effect, which is the main focus of this subsection.

Photostriction in ferroelectric materials was studied as early as in 1966 in SbSI crystals, with a $004 \%$ change in the length of the polar axis under white light illumination [13]. An order of magnitude larger effect was observed later on by Xiao et al. in Fe-doped lithium niobate crystals [14]. Interestingly, the photostrictive properties of ferroelectrics have mostly been interpreted as being driven by their piezoelectric properties (strain linearly induced by a change in polarization or electric field) [15]. As a result, investigations of materials with large piezoelectric properties, such as the ones usually found in so-called Morphotropic Phase Boundaries (MPBs), were at the forefront of research in the 90's. In particular, $\left(\mathrm{Pb}_{1-x} \mathrm{La}_{x}\right)\left(\mathrm{Zr}_{1-y} \mathrm{Ti}_{y}\right) \mathrm{O}_{3}$ (PLZT) was comprehensively studied $[16,17,18]$, and devices based on the photostriction effect and PLZT were 
designed to take advantage of its large coupling of light and strain [19]. However, most classical ferroelectric materials, including PLZT, have large bandgaps that often rise above $3 \mathrm{eV}$ [20], which lies at best at the extreme side of the visible range, and most often, in the ultraviolet (UV) domain. As a result, research on the conversion of solar energy into mechanical energy (but also into electrical energy) using ferroelectrics has been at a standstill for a decade or so. Recently though, new prospects have appeared in the field, mainly driven by the appearance of low band-gap FE and MFE materials. For instance, Nechache et al. have achieved a low band-gap of $1.5 \mathrm{eV}$ in multiferroic $\mathrm{Bi}_{2} \mathrm{FeCrO}_{3}$, eventually resulting in one of the most efficient perovskite oxide-based solar cell [4]. Most recent photostriction experiments have been conducted on multiferroic bismuth ferrite, whose band-gap ranges from 2.2 to $2.7 \mathrm{eV}[2,21]$, and is therefore suitable for visible light absorption. As of right now, photostriction of $0.004 \%$ was reported in bismuth ferrite $[22,15]$. The photostriction effect in bismuth ferrite has recently been used to increase the coercivity of a Nickel film deposited on a $\mathrm{BiFeO}_{3}$ single crystal by $50 \%$ [23], opening the way to strain-mediated optical control of magnetic properties. Note also that the photostriction effects described so far are taking place at the scale of seconds or even minutes in the case of lithium niobate [14]. On the other hand, pump-probe experiments have demonstrated the occurence of photo-generated strain fields at the scale of picoseconds and below $[24,25,26,27,28,29,30,31]$. Interestingly, Ref. [27] showed the possibility, depending on the orientation of $\mathrm{BiFeO}_{3}$ grains with respect to the probe, to generate preferentially shear or longitudinal acoustic waves.

There is a variety of mechanisms contributing to photostriction [32, 33, 34]. First, light-matter interaction, through the interaction of photoexcited carriers with the lattice, creates heating of the material, resulting in thermal dilation. Second, the electronic density redistribution generated by the excitation causes lattice to deform through a mechanism known as the deformation potential $[35,36,34]$ (often termed electronic pressure as well). Third, in materials lacking inversion symmetry, such as ferroelectrics, the converse piezoelectric effect caused by the appearance of a photo-induced electric field results in the appearance of a photo-induced deformation [15, 32, 34]. The latter mechanism is particularly appealing, as it provides polar materials (and ferroelectric materials in particular) with an additional handle compared to centrosymmetric materials. In addition, it is known how to design large piezoelectric and ferroelectric materials, by mixing a ferroelectric material (such as lead titanate) with a relaxor or antiferroelectric material (such as lead magnesium niobate or lead zirconate). Such mixing results in the appearance of low symmetry (often monoclinic, sometimes orthorhombic) phases for a specific compositional range defining the aforementionned MPB [37, 38, 39, 40]. The existence of these lowsymmetry phases in the phase diagram has been demonstrated to host giant piezoelectric properties [41].

The origin of photostriction can be qualitatively captured using a simple Landau model $[15,42]$. Consider a uniaxial ferroelectric material. Its thermodynamic potential $\Phi_{F}(T, \boldsymbol{P}, \boldsymbol{\eta})$, in the dark, can be expressed as a Taylor expansion in terms of the order parameters (polarization $\boldsymbol{P}$ and strain $\boldsymbol{\eta}$ ) with 
respect to the reference paraelectric phase,

$$
\begin{aligned}
\Phi_{F}(T, \boldsymbol{P}, \boldsymbol{\eta}) & =\Phi_{P}(T)+\frac{\alpha(T)}{2} \boldsymbol{P}^{2}+\frac{\beta}{4} \boldsymbol{P}^{4} \\
& +\quad+\frac{\gamma}{6} \boldsymbol{P}^{6}+\frac{C}{2} \boldsymbol{\eta}^{2}+\frac{Q}{2} \boldsymbol{P}^{2} \boldsymbol{\eta}
\end{aligned}
$$

In this equation, $\alpha(T)$ is a second rank tensor. $\beta$ and $\gamma$ are fourth and sixth rank tensors, which are considered temperature independent. $Q$ is the fourth-rank electrostrictive tensor, and $C$ is the second-rank stiffness tensor. At equilibrium, the thermodynamic potential must reach a stationary point, and thus the following set of equations must be solved,

$$
\begin{aligned}
& \frac{\partial \Phi_{F}}{\partial \boldsymbol{P}}=0=\alpha(T) \boldsymbol{P}+\beta \boldsymbol{P}^{3}+\gamma \boldsymbol{P}^{5}+Q \boldsymbol{P} \boldsymbol{\eta}, \\
& \frac{\partial \Phi_{F}}{\partial \boldsymbol{\eta}}=0=C \boldsymbol{\eta}+\frac{1}{2} Q \boldsymbol{P}^{2} .
\end{aligned}
$$

The perturbation caused by illumination can be considered as adding the energy of the photo-excited electrons and substracting those of the holes [15], in a similar fashion to what has been done to study heavily doped ferroelectric materials [43]. As a result, the thermodynamic potential of the gas of photoexcited electrons and holes must be considered alongside that of Eq. (1). The total thermodynamic potential is therefore

$$
\Phi(T, \boldsymbol{P}, \boldsymbol{\eta})=\Phi_{F}(T, \boldsymbol{P}, \boldsymbol{\eta})+\Phi_{p h}(T, \boldsymbol{P}, \boldsymbol{\eta})
$$

with

$$
\Phi_{p h}(T, \boldsymbol{P}, \boldsymbol{\eta})=\sum_{n, \boldsymbol{k}} \delta n_{n \boldsymbol{k}} \varepsilon_{n \boldsymbol{k}} .
$$

In Eq. (5), $\delta n_{n \boldsymbol{k}}$ is the change of occupancy of the Bloch state $n \boldsymbol{k}$ with respect to the system in the dark, and $\varepsilon_{n \boldsymbol{k}}$ is the energy of that state. Since photon absorption creates the same amount of holes and electrons, the condition $\sum_{n, \boldsymbol{k}} \delta n_{n \boldsymbol{k}}=0$ must be satisfied. In the simple case of a two-level system, for which holes are created at the top of valence band $\varepsilon_{v}$ while electrons are photoexcited at the bottom of the conduction band $\varepsilon_{c}$, the former equation can be simply written as,

$$
\Phi_{p h}(T, \boldsymbol{P}, \boldsymbol{\eta})=\delta n_{e} E_{v \rightarrow c},
$$

with $\delta n_{e}$ being the number of photoexcited electrons in the conduction band, and $E_{v \rightarrow c}=\varepsilon_{c}-\varepsilon_{v}$. The latter is a function of temperature, polarization and strain. Assuming that the perturbation introduced by switching on the light is small, the order parameters can be expanded around their equilibrium in the dark. That is, they can be written $\boldsymbol{P}=\boldsymbol{P}_{0}+\delta \boldsymbol{P}$ and $\boldsymbol{\eta}=\boldsymbol{\eta}_{0}+\delta \boldsymbol{\eta}$, with the 
quantities having the subscript " 0 " referring to the equilibrium values in the dark, which are solutions of Eqs. (2) \& (3). It is then straightforward to define, as done in Ref. [42], a photo-induced stress $\boldsymbol{\sigma}_{\text {photo }}$ and photo-induced electric field $\boldsymbol{E}_{\text {photo }}$,

$$
\begin{aligned}
\boldsymbol{\sigma}_{\text {photo }} & =-\left.\delta n_{e} \frac{\partial E_{v \rightarrow c}}{\partial \boldsymbol{\eta}}\right|_{\boldsymbol{P}_{0}, \boldsymbol{\eta}_{0}}, \\
\boldsymbol{E}_{\text {photo }} & =-\left.\delta n_{e} \frac{\partial E_{v \rightarrow c}}{\partial \boldsymbol{P}}\right|_{\boldsymbol{P}_{0}, \boldsymbol{\eta}_{0}},
\end{aligned}
$$

allowing to re-write Eq. (6) as

$$
\Phi_{p h}(T, \boldsymbol{P}, \boldsymbol{\eta})=\Phi_{p h}\left(T, \boldsymbol{P}_{0}, \boldsymbol{\eta}_{0}\right)-\boldsymbol{\sigma}_{p h o t o} . \delta \boldsymbol{\eta}-\boldsymbol{E}_{p h o t o} . \delta \boldsymbol{P} .
$$

As a result, the equilibrium conditions under illumination, in this simple model, are

$$
\begin{aligned}
& \frac{\partial \Phi}{\partial \boldsymbol{P}}=0=\alpha(T) \boldsymbol{P}+\beta \boldsymbol{P}^{3}+\gamma \boldsymbol{P}^{5}+Q \boldsymbol{\eta} \boldsymbol{P}-\boldsymbol{E}_{p h o t o} \\
& \frac{\partial \Phi}{\partial \boldsymbol{\eta}} \quad=0=C \boldsymbol{\eta}+\frac{1}{2} Q \boldsymbol{P}^{2}-\boldsymbol{\sigma}_{\text {photo }},
\end{aligned}
$$

which, under the assumption that $\delta \boldsymbol{P}$ and $\delta \boldsymbol{\eta}$ are indeed small compared to the equilibrium values in the dark, can be linearized, leading to [42]

$$
\begin{aligned}
\delta \boldsymbol{P} & =\chi_{p h} \boldsymbol{E}_{\text {photo }}+d_{p h}^{T} \boldsymbol{\sigma}_{\text {photo }} \\
\delta \boldsymbol{\eta} & =S_{p h} \boldsymbol{\sigma}_{\text {photo }}+d_{p h} \boldsymbol{E}_{\text {photo }}
\end{aligned}
$$

In those latter equations, $\chi_{p h}$ is the dielectric susceptibility under illumination, $d_{p h}$ is the piezoelectric constant under illumination, and $S_{p h}$ the elastic compliance under illumination.

From Eq. (13), the contribution of the potential deformation mechanis$\mathrm{m}$ (through the photo-induced stress $\boldsymbol{\sigma}_{\text {photo }}$ ) and the piezoelectric mechanism (caused by the photo-induced electric field $\boldsymbol{E}_{\text {photo }}$ ) appear naturally. To be exhaustive, one should also include the contribution of lattice heating and thermal dilatation. This can be done by adding the energy of the lattice/phonon gas, $\Phi_{L}=\sum_{n, \boldsymbol{q}} \delta n_{p h, n \boldsymbol{q}} \hbar \omega_{n \boldsymbol{q}}$, in which $\delta n_{p h, n \boldsymbol{q}}$ and $\hbar \omega_{n \boldsymbol{q}}$ represent the change in population and energy of the $n^{t h}$ phonon mode at wavevector $\boldsymbol{q}$ [33].

\subsection{Elasto-optic effect}

Elasto-optic effect describes the change of permittivity, $\varepsilon$, due to mechanical strain, $\eta$. It can be used for creating or designing acousto-optic modulators, 
acousto-optic tunable filter, electro-optic deflection, etc [44]. Such effect can be quantified by the elasto-optic tensor, $p_{i j}$, that is defined via

$$
\Delta\left(\frac{1}{\tilde{\varepsilon}}\right)_{i}=\sum_{j} p_{i j} \eta_{j}
$$

where $i$ are $j$ are both integers that can vary from 1 to 6 (when adopting the Voigt notation). Introducing the extinction coefficient $k$ and refractive index $n$ tensors via

$$
\tilde{\varepsilon}=\varepsilon^{\prime}+i \varepsilon^{\prime \prime}=(n+i k)^{2},
$$

Under low-frequency field (the Pockels effect), $\varepsilon^{\prime \prime}$ is about zero, and Eq. (14) can be rewritten as

$$
\Delta\left(\frac{1}{n^{2}}\right)_{i}=\sum_{i} p_{i j} \eta_{j} .
$$

Eq. (16) was used in Ref. [45] to compute the elasto-optic coefficient. Note that, as indicated above, both refractive index $n$ and strain $\eta$ have 6 components when using the Voigt notation, implying that there are 36 components for the elastooptic coefficient $p_{i j}$. However, common elasto-optic materials (such as $\mathrm{SiO}_{2}$ and $\mathrm{LiNbO}_{3}$ ) have relatively high symmetry, and, as a result, only a few of the elasto-optic tensor components are finite.

\section{Methods}

\subsection{Photostriction using the $\triangle \mathrm{SCF}$ method}

When performing Density Functional Theory (DFT) calculations, one is typically minimizing the energy functional with respect to density by self-consistently resolving the ground state density, in accordance with the two Hohenberg-Kohn theorems at the foundation of DFT [46]. There are several schemes to achieve such calculations, however one simple, popular and practical way is to assume that one can find a system of non-interacting electrons with the same energy and ground state density as the true system of fully interacting electrons. Such approximation is called the Kohn-Sham (KS) ansatz [47], and provides a physi-

cally intuitive one-electron picture, within which one is left to fill monoelectronic orbital states (determined from a monoelectronic Schrödinger equation) by increasing levels of energy. As described in Fig. (1), a KS calculation would start by guessing an input potential $V_{i n}$, thereby fully determining the one-electron Hamiltonian. This Hamiltonian is then solved, resulting in a set of monoelectronic orbitals $\varphi_{i}$ with eigenvalues $\varepsilon_{i}$. The lowest energy lying states would then be filled in order to determine the ground state, and the new potential $V_{\text {out }}$ generated by the distribution of electrons in these occupied orbitals becomes the input for a new cycle, until convergence of the ground state density is reached up to a desired accuracy. 


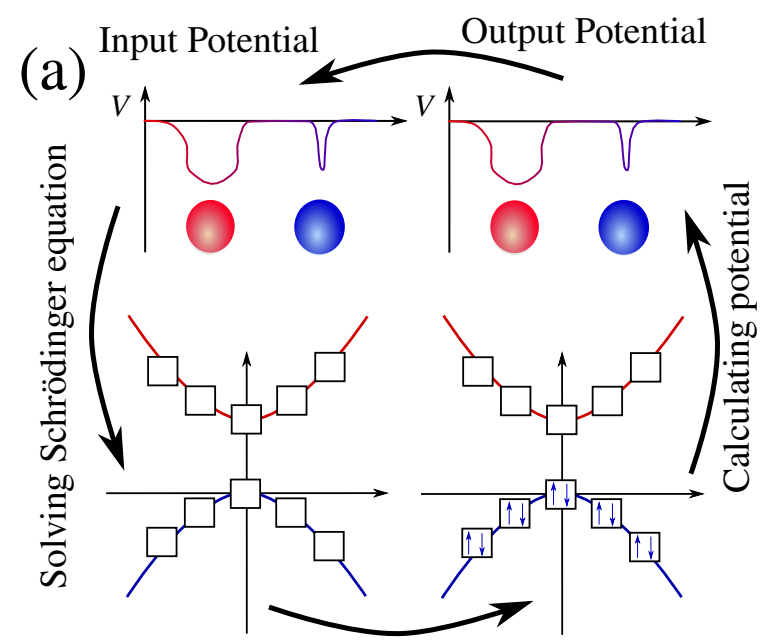

Filling KS orbitals

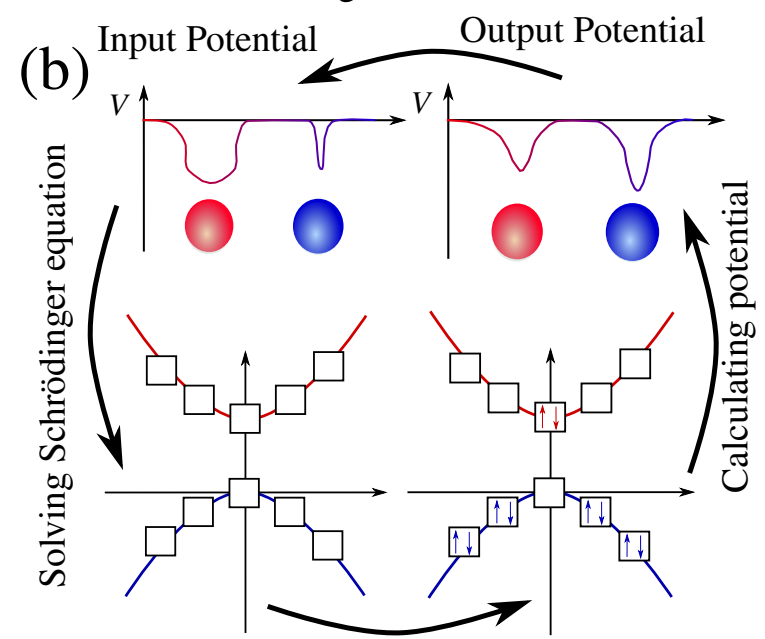

Filling KS orbitals under constraints

Figure 1: (a) Regular Kohn-Sham calculation to find the ground state electronic density: from an input potential $V_{i n}$, the now known Kohn-Sham Hamiltonian can be diagonalized. Its lowest eigenstates ("orbitals") are then filled with electrons, and a new potential is calculated based on the latter electronic distribution. The output potential is then plugged in as input potential until convergence to a reasonable accuracy is reached. (b) $\triangle \mathrm{SCF}$ calculation: at filling, not all low energy lying eigenstates are filled, but some electrons are imposed to occupy excited orbitals. An output potential is then calculated based on this electronic distribution, and the density is self-consistently solved under the constraint of certain occupation numbers. Reproduced from Ref. [42]. Copyright 2017 American Physical Society. 
The $\Delta$ SCF method [48] is essentially analog to a regular Kohn-Sham calculations: starting from an input potential $V_{i n}$, the monoelectronic Schrödinger equation is solved. The main difference arises when the filling of the orbitals is performed. As sketched in Fig. (1.b), some electrons are constrained to occupy excited states in the conduction band, while leaving holes in the valence band. Therefore, the system is converged under the constraint of having some electrons lying in higher energy states, and holes in lower energy states. This rather simple approach has been proven to be rather successful in describing resonance level in finite systems such as molecules adsorbed on metallic surfaces [49], or the ligand-field splitting in Fe-phtalocyanine [50]. In infinite systems however, the $\triangle \mathrm{SCF}$ method does not improve much the description of excited states energies such as the bandgap energy when using traditional functionals [51]. One should resort to more accurate models, such as the $G W$ approximation [52] or Time Dependent DFT (TD-DFT) [53], which are rather computationally expensive. Performing structural relaxation with the former technique is even practically untractable. Furthermore, we note that many $G W$ calculations are typically used as corrections to eigenenergies and wavefunctions determined with a more traditional functional [54]. As a result, the $\triangle \mathrm{SCF}$ method is a useful tool to apprehend photostrictive phenomena.

During $\triangle \mathrm{SCF}$ calculations, a $k$-point $A$ belonging to the valence band is emptied, meanwhile a $k$-point $B$ belonging to the conduction band is filled with electrons, mimicking what we call a $A \longrightarrow B$ transition. In the following works described below, only cases for which the photoexcited holes (electrons resp.) are sitting at the top (bottom) of the valence (conduction) band are considered. This amounts to assume that electron-phonon scattering, responsible for the thermalization of the photo-excited carriers, is extremely fast compared to other processes such as optical recombination of electrons with holes, the latter typically ranging in the nano- to microsecond according to Ref. [33], while electron-phonon thermalization processes typically occur on a picosecond time scale. In other words, the influence of the non-thermalized electrons, which are known to be responsible for ballistic photovoltaic current in ferroelectric$\mathrm{s}[8,10,11]$, is neglected.

\subsection{Elasto-optic coefficients}

First-principle techniques were used in Ref. [45] to calculate the frequencydependent dielectric tensor once the electronic ground state was determined. More precisely, the imaginary part of dielectric function was computed via the following equation [55]

$$
\begin{aligned}
\varepsilon^{\prime \prime}(\omega)= & \frac{4 \pi^{2} e^{2}}{\Omega} \lim _{q \rightarrow 0} \frac{1}{q^{2}} \sum_{c, v, \mathbf{k}} 2 \omega_{\mathbf{k}} \delta\left(\epsilon_{c \mathbf{k}}-\epsilon_{v \mathbf{k}}-\hbar \omega\right) \\
& \times<u_{c \mathbf{k}+\mathbf{e}_{\alpha} \mathbf{q}}\left|u_{v \mathbf{k}}><u_{c \mathbf{k}+\mathbf{e}_{\beta} \mathbf{q}}\right| u_{v \mathbf{k}}>^{*},
\end{aligned}
$$


where $c$ and $v$ refer to band indices of the conduction and valence bands, respectively. $u_{c \mathbf{k}}$ is the cell periodic part of the Bloch orbitals at the reciprocal space point $\mathbf{k} ; \mathbf{e}_{\alpha}$ and $\mathbf{e}_{\beta}$ are unit vectors along the $\alpha$ and $\beta$ Cartesian direction, respectively; and the asterisk symbol denotes complex conjugate. The real part of the dielectric tensor $\varepsilon_{\alpha \beta}^{\prime}$ is then extracted through the Kramers-Kronig transformation

$$
\varepsilon^{\prime}(\omega)=1+\frac{2}{\pi} P \int_{0}^{\infty} \frac{\varepsilon_{\alpha \beta}^{\prime \prime}\left(\omega^{\prime}\right) \omega^{\prime}}{\omega^{\prime 2}-\omega^{2}} d \omega^{\prime},
$$

where $P$ denotes the so-called principal value [55].

In the low frequency range, complex refractive index, with real part $(n)$ and imaginary part $(k)$, and complex dielectric function, with real part $\left(\varepsilon^{\prime}\right)$ and imaginary $\left(\varepsilon^{\prime \prime}\right)$, follow the relation

$$
\tilde{\varepsilon}=\varepsilon^{\prime}+i \varepsilon^{\prime \prime}=\tilde{n}^{2}=(n+i k)^{2}
$$

Reference [45] concentrated on energies $\hbar \omega^{\prime}$ smaller than the electronic band gap, which implies that, as consistent with the delta function appearing in Eq.(17), $\varepsilon^{\prime \prime}(\omega)$ vanishes for the selected energies. The refractive index is thus simply equal to the square root of $\varepsilon^{\prime}(\omega)$. Our first-principles computations of the refractive index within its band gap are found to be rather accurate. This is demonstrated by the prediction in Ref. [45] of an average refractive index, $\left(n_{11}+n_{22}+n_{33}\right) / 3$ of 2.8 for $\mathrm{PbTiO}_{3}$ for an energy about $40 \%$ smaller than the band gap, which compares well with the measured value of 2.7 at a wavelength of $633 \mathrm{~nm}$.

Reference [45] then computed the elasto-optic coefficient, $p_{i j}$, via the aforementionned Eq. (16). Only the ' 1 ' and '2' components of the strain tensor (with the ' 1 ', ' 2 ' and ' 3 ' axes being along the pseudo-cubic [100], [010] and [001] directions, respectively) were considered in this formula, with $\eta_{1}=\eta_{2}$, to reflect epitaxial growth of the film. The resulting effective elasto-optic coefficients are thus obtained from the following relations: $1 /\left(n_{1}^{2}\left(\eta_{1}\right)\right)-1 /\left(n_{1}^{2}(0)\right)=\left(p_{11}+p_{12}\right) \eta_{1}$, $1 /\left(n_{2}^{2}\left(\eta_{1}\right)\right)-1 /\left(n_{2}^{2}(0)\right)=\left(p_{21}+p_{22}\right) \eta_{1}$, and $1 /\left(n_{3}^{2}\left(\eta_{1}\right)\right)-1 /\left(n_{3}^{2}(0)\right)=\left(p_{31}+\right.$ $\left.p_{32}\right) \eta_{1}$. Note that for the tetragonal, orthorhombic, rhombohedral and the monoclinic $C m$ phases of epitaxial $\mathrm{PbTiO}_{3}$ films (see Section 4 for a description of these phases), $n_{1}=n_{2}=n_{o}$, which is known as the ordinary refractive index, and $n_{3}=n_{e}$, which is termed the extraordinary refractive index. Note that first-principles methods can predict elasto-optic coefficients accurately. For example, for bulk $\mathrm{LiNbO}_{3}$ (using the hexagonal setting), one gets $p_{31}=0.17$ for a photon energy being roughly half-way within its band gap [45], which is in very good agreement with the corresponding experimental value of 0.18 [56]. 


\section{Modelling photostriction in multiferroic and ferroelectric materials}

\subsection{Photostriction in classical ferroelectric materials}

Among the vast family of ferroelectric materials, barium and lead titanate emerge as prototypical examples. Both are perovskite oxides, with barium or lead ions occupying the A-sites of the perovskite structure while titanium ions sit on the B-sites. Barium titanate undergoes three successive phase transitions, from the high-symmetry $P m \overline{3} m$ paraelectric phase at high temperature to a ferroelectric tetragonal $P 4 \mathrm{~mm}$ phase at $390 \mathrm{~K}$, before transforming to an orthorhombic ferroelectric phase around $280 \mathrm{~K}$, and eventually goes into a ferroelectric rhombohedral $R 3 \mathrm{~m}$ phase below $180 \mathrm{~K}$ [57]. Lead titanate is even simpler, as it experiences only one transition from the high-symmetry cubic $P m \overline{3} m$ phase to a ferroelectric tetragonal $P 4 m m$ phase at $766 \mathrm{~K}[1]$.

Both compounds have been successfully described in first-principles calculations using the Local Density Approximation (LDA) functional, and this was the starting point of Ref. [42], in which other technical details are provided. We describe here only the photostriction induced in the ground state of those compounds, that is the $R 3 \mathrm{~m}$ and $P 4 \mathrm{~mm}$ phases of barium and lead titanate respectively. The band structure of both compounds are presented in Fig. (2). The considered "transitions" are $Z \rightarrow Z, Z \rightarrow \Gamma, X \rightarrow \Gamma$ and $X \rightarrow Z$ for $\mathrm{PbTiO}_{3}$ (PTO) and $Z \rightarrow \Gamma$ and $\Gamma \rightarrow \Gamma$ for rhombohedral $\mathrm{BaTiO}_{3}$ (BTO). Again, the assumption is made that the thermalization of the phonons is sufficiently fast as compared to the photoexcitation and recombination processes to consider that the hole and electrons are located at the top and bottom of valleys in the valence and conduction bands. According to Ref. [33], the relaxation time of the electron to the bottom of the conduction band is typically of the order of $1 \mathrm{ps}$, while recombination has a much larger (1 ns- $1 \mu \mathrm{s})$ relaxation time, justifying this approximation. Note also that the first-principle description of ultra-fast phenomena such as those occuring in Refs. [25, 26, 28, 29] is out of the scope of this review because of the above mentioned approximation.

The results obtained for the aforementioned transitions in $\mathrm{PbTiO}_{3}$ are presented as filled symbols in Fig. (3). Because of the same $d_{x^{2}-y^{2}}$ character of the conduction states at $\Gamma$ and $X$ [see Fig. (4) in Ref. [42]], the results are insensitive to the location of the electrons in $\Gamma-Z$ branch of the conduction band. This can be seen as transitions involving electrons at $\Gamma$ or $Z$ show the same behavior, provided the hole is located at the same point in the valence band. When the hole sits at $X$ in the valence band, the change of lattice constant in the polar direction is small compared to that induced when the hole sits at $Z$. In the direction normal to the polar axis (left panel of Fig. (3)), the hole sitting at $Z$ generates a significant expansion, while when located at $X$, only a minor reduction is found. Note that at $X$ (which is the top of the valence band), the hole has a combined $\mathrm{Pb} 6 s$ and $\mathrm{O} 2 p_{x}$ character, while at $Z$ it has $\mathrm{Pb} 6 s$ and $\mathrm{O}$ $2 p_{z}$ character [42]. 

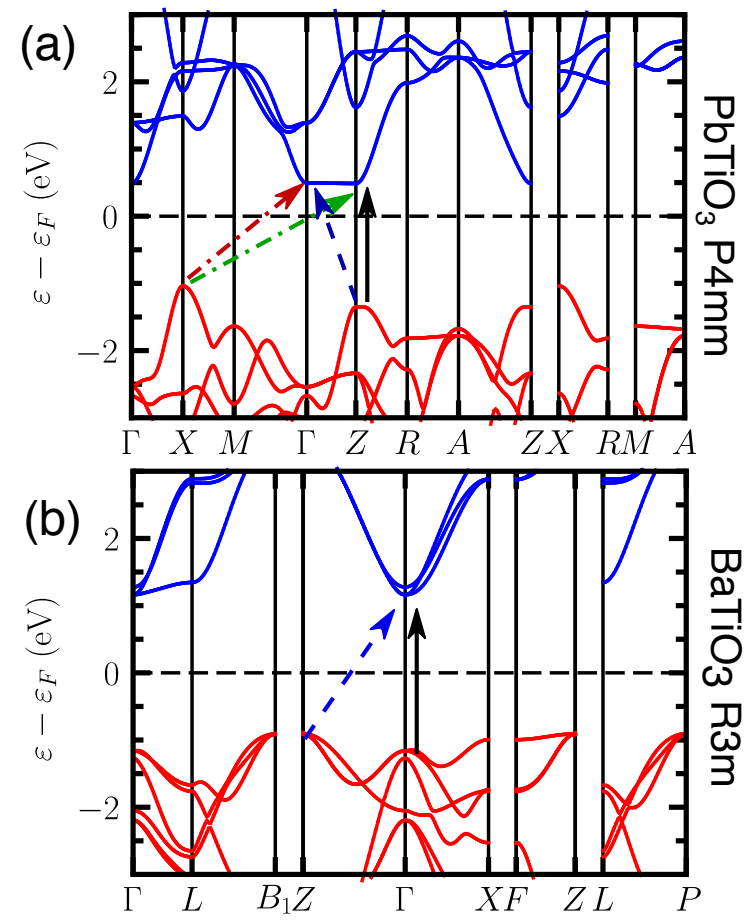

Figure 2: Band structures of (a) tetragonal lead titanate, and (b) rhombohedral barium titanate. The reference energy is placed at the middle of the Kohn-Sham gap. The arrows represent the different transitions that are considered. Adapted from Ref. [42]. Copyright 2017 of the American Physical Society. 


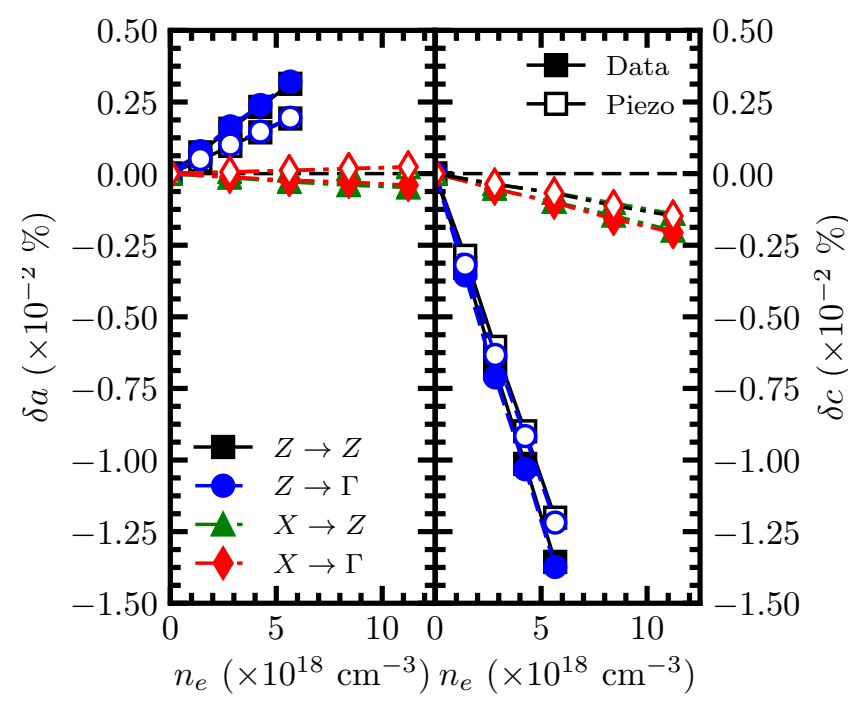

Figure 3: Change of lattice constant orthogonal (left panel) and parallel (right panel) to the tetragonal axis in the $P 4 \mathrm{~mm}$ phase of lead titanate, as obtained from $\triangle \mathrm{SCF}$ calculations (filled symbols) and as estimated from the converse piezoelectric model (open symbols). Reproduced from Ref. [42]. Copyright 2017 from the American Physical Society. 
As described in Section 1.2, photostriction in polar materials can be understood as originating from three main contributions: potential deformation, converse piezoelectric effect following a photo-induced electric field, and thermal dilatation following photo-induced heating. During $\triangle \mathrm{SCF}$ calculations, the temperature is $0 \mathrm{~K}$. As a result, thermal dilatation is not taken into account in those calculations. We now address the question of which of the two former mechanisms, between potential deformation and piezoelectric effect, is the dominant mechanism.

First, it is noted that the ionic relaxation can be translated into a change of polarization through the Born approximation,

$$
\delta P_{i}=\frac{e}{V_{0}} \sum_{\alpha, j} Z_{i j}^{\alpha \star} \delta u_{j}^{\alpha} .
$$

In Eq. (20), $e$ is the elementary electric charge, $\alpha$ denotes the atom number in the unit cell, $\delta u_{j}^{\alpha}$ its displacement in the $j$-th direction with respect to a reference configuration (here the ground-state structure), and $Z_{i j}^{\alpha \star}$ is the Born effective charge tensor of atom $\alpha$. Of course, a major approximation is made here: it is assumed that the Born effective charge does not change between the ground state and the excited state, which is, in principle, questionable. However, as discussed in Ref. [42], because the amount of excited charges is quite small, this approximation seems reasonable. The change of polarization in lead titanate, using Born charges calculated with Density Functional Perturbation Theory (DFPT) [42], is depicted in the left panel of Fig. (4). For all considered transitions, the polarization decreases. The strongest decrease rate happens for the $Z \longrightarrow Z$ and $Z \longrightarrow \Gamma$ transitions, and also correspond to the strongest photostriction effect calculated in Fig. (3).

This change in polarization can be directly related to a change of strain state by the third rank converse piezoelectric tensor $\boldsymbol{g}$,

$$
\delta \boldsymbol{\eta}=\boldsymbol{g} . \delta \boldsymbol{P} .
$$

The converse piezoelectric tensor $\boldsymbol{g}$ is trivially related to more well-known piezoelectric tensor $\boldsymbol{d}$ (which relates the change of strain to an electric field) by $\boldsymbol{g}=\boldsymbol{d} \cdot \boldsymbol{\chi}^{-1}$, with $\chi$ being the dielectric susceptibility. After calculating the piezoelectric tensor from DFPT calculations [42], the piezoelectric-induced change of lattice constant, as estimated from Eq. (21) and Fig. (4), was plotted as open symbols in Fig. (3). It can be observed there that along the polar axis, the piezoelectric model accounts for most of the calculated effect, for all transitions. In the direction perpendicular axis, the situation is not as clear. On one hand, the calculated results agree well with the piezoelectric model for the $Z \longrightarrow Z$ and $Z \longrightarrow \Gamma$ transitions, which are the ones with the strongest change of polarization. On the other hand, due to the negative value of the $g_{13}$ component, a weak expansion of the lattice is predicted in the plane orthogonal to the polar axis from the converse piezoelectric model for the two transitions departing from the $X$ point (for which the induced change in polarization is weaker) while the $\triangle \mathrm{SCF}$ data rather shows a small contraction. This latter 


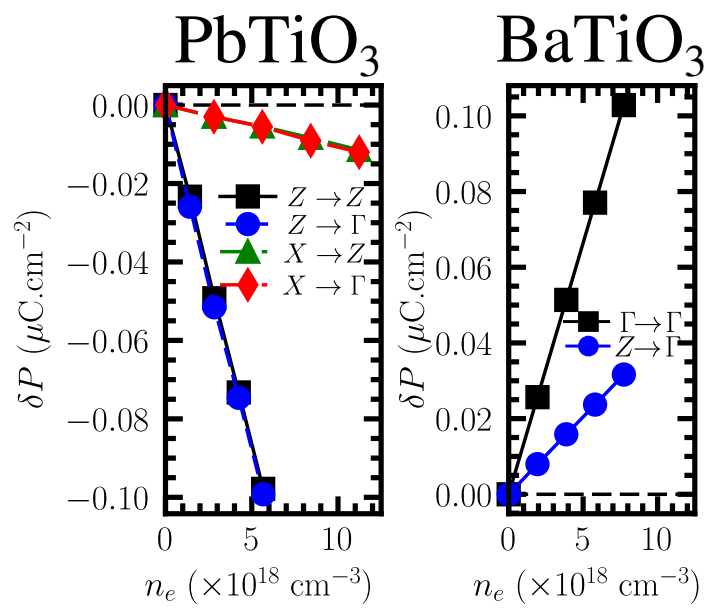

Figure 4: Change of polarization against the concentration of excited carriers, for different transitions in (left) tetragonal lead titanate and (right) rhombohedral barium titanate. Reproduced from Ref. [42]. Copyright 2017 American Physical Society.

difference might result from the action of a different mechanism, for instance the potential deformation (also sometimes coined electronic pressure).

To check whether the potential was of any influence in the obtained results, $\triangle \mathrm{SCF}$ calculations were performed at fixed atomic positions. In other words, only the volume of the cell is allowed to relax, and thus the polarization is kept approximately constant (up to the change of volume and the minor electronic dipole induced [42]). The results, plotted as open blue circles in Fig. (5) in the case of a $Z \longrightarrow Z$ transition in $\mathrm{PTO}$, show that along the polar axis the potential deformation mechanism is a negligible contribution compared to the piezoelectric effect. The picture is however different in the directions perpendicular to the polarization, for which the associated piezoelectric constant is smaller. It is found then that the potential deformation contribution could account for as much as a third of the calculated effect. More importantly, in the case of the $X \longrightarrow Z$ transition, although the electronic pressure mechanism brings again a small contribution to the photostriction of the polar axis, it actually now competes and overcomes the (small) expansion caused by the piezoelectric effect, and results in an overall contraction of the $a$ axis [see Fig. (6)]. Because the electronic pressure competes with the converse piezoelectric effect in the case of the $X \longrightarrow Z$ transition, the $c$ axis contraction is also reduced compared to that found in the $Z \longrightarrow Z$ transition [see Fig. (6.b)]. Actually, observing Fig. (6.b), the $\triangle S C F$ data are found to lead to a larger contraction than predicted using the piezoelectric model, despite the competition of the electronic pressure mechanism. It must however be noted that both the piezoelectric model and frozen ion $\triangle S C F$ calculations can only be taken as qualitative estimates of the 


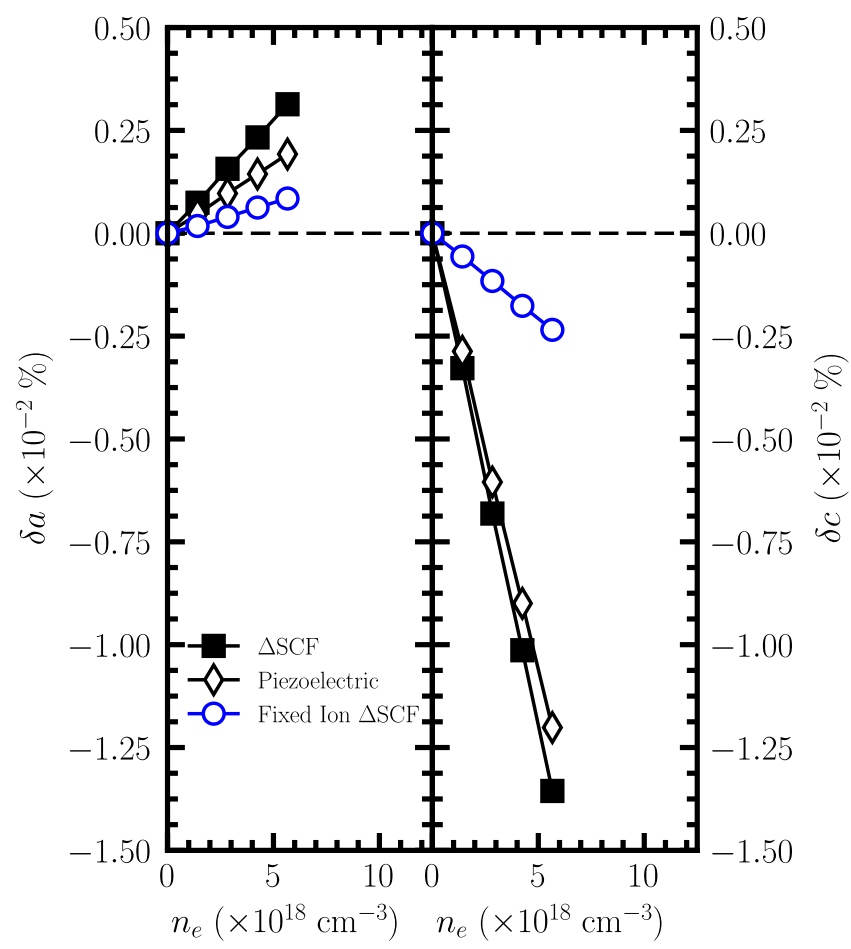

Figure 5: Photo-induced change of lattice constant in tetragonal $\mathrm{PbTiO}_{3}$ for a $Z \longrightarrow Z$ transition, as calculated from a fully relaxed $\Delta \mathrm{SCF}$ calculation (black squares), as estimated from the piezoelectric model (open black diamonds) and as calculated using frozen atomic position calculations (open blue circles), for the polar axis direction (right panel) and the direction perpendicular to it (left panel). Adapted from Ref. [42].

importance of the piezoelectric and potential deformation contributions to photostriction, as they rely on quite strong assumptions. Typically, we compute the change of polarization necessary to evaluate the converse piezoelectric effect by using ground state Born charges and finite displacement. As a result, it is most likely that only orders of magnitude and qualitative trends may be safely discussed when decomposing the photostriction effect in terms of those basic mechanisms.

In the case of barium titanate, depicted in Fig. (7), the pseudo-cubic lattice constant is found to decrease with increasing concentration of photoexcited for both the $\Gamma \longrightarrow \Gamma$ and the $Z \longrightarrow \Gamma$. Meanwhile, the pseudocubic angle slightly increases. A similar analysis in terms of piezoelectric and electronic pressure source terms for the calculated photostriction was conducted in Ref. [42]. It was shown there that the piezoelectric effect was the dominant mechanism in the 


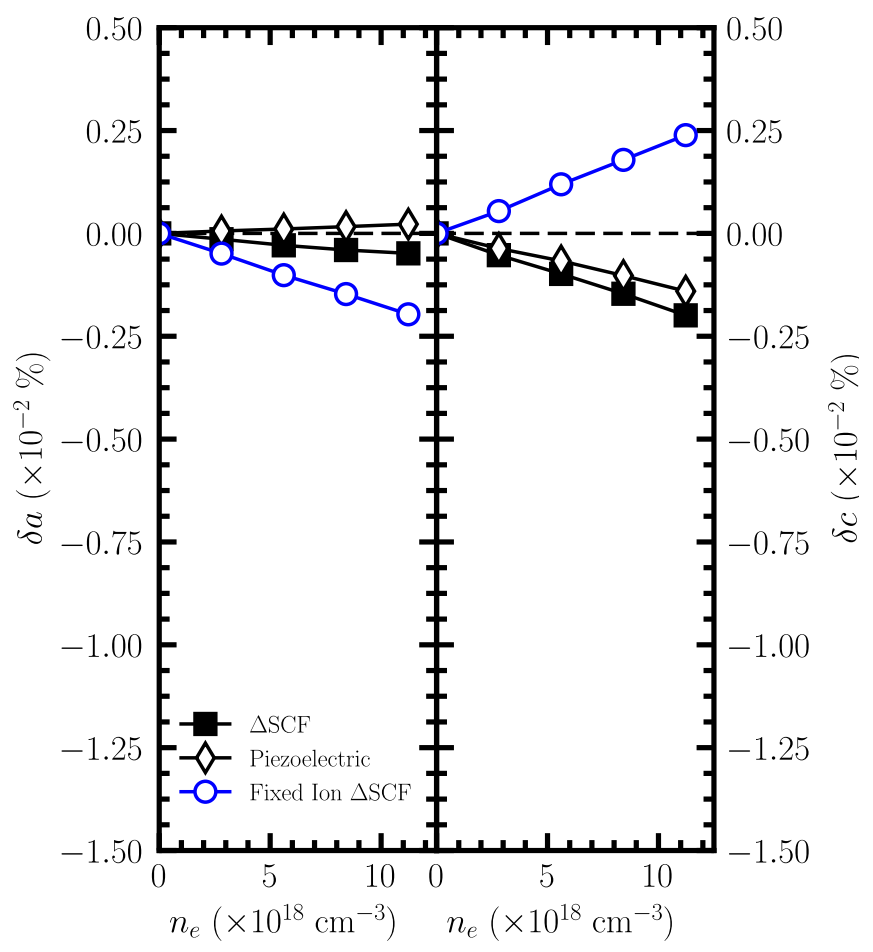

Figure 6: Photo-induced change of lattice constant in tetragonal $\mathrm{PbTiO}_{3}$ for a $X \longrightarrow Z$ transition, as calculated from a fully relaxed $\Delta$ SCF calculation (black squares), as estimated from the piezoelectric model (open black diamonds) and as calculated using frozen atomic position calculations (open blue circles), for the polar axis direction (right panel) and the direction perpendicular to it (left panel).Adapted from Ref. [42]. 


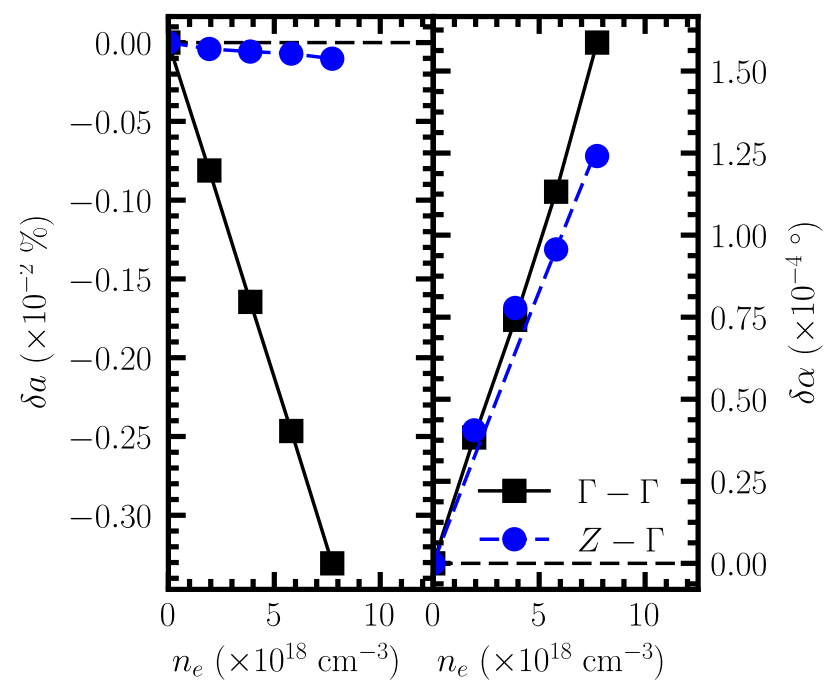

Figure 7: Change of pseudo-cubic lattice constant (left panel) and pseudocubic angle (right panel) versus concentration of excited electrons for different transitions in rhombohedral barium titanate. Reproduced from Ref. [42]. Copyright 2017 American Physical Society.

case of the $\Gamma \longrightarrow \Gamma$ transition, but that potential deformation actually competes with the photo-induced piezoelectric effect for the $Z \longrightarrow \Gamma$ transition.

\subsection{Photostriction in bismuth ferrite}

Bismuth ferrite has a rhombohedral structure with space group $R 3 c$ below $1103 \mathrm{~K} \mathrm{[2]}$, whose unit cell is sketched in green in Fig. (8). It is made of two pseudocubic 5-atom perovskite cells (depicted as black cubes in Fig. (8)) along the $[111]_{p c}$ pseudocubic direction because of the $a^{-} a^{-} a^{-}$tilting pattern (in Glazer notation [58]) of the oxygen octahedra $[59,60,61]$. The [111] $]_{p c}$ direction is also associated with a large polarization of about $100 \mu \mathrm{C} \cdot \mathrm{cm}^{-2}[62,63]$. $\mathrm{BiFeO}_{3}(\mathrm{BFO})$ also exhibits a $G$-type antiferromagnetic order convoluted with cycloidal arrangement of the antiferromagnetic vector, propagating in the $[1 \overline{1} 0]_{p c}$ whilst the antiferromagnetic vector rotates in the plane spanned by $[1 \overline{1} 0]_{p c}$ and $[111]_{p c}$ with a period of $62-64 \mathrm{~nm}[64,65,66,2]$.

$\mathrm{BiFeO}_{3}$, among ferroelectrics and multiferroics, has a relatively low bandgap, estimated to be of the order of $2.7 \mathrm{eV}$ [67]. However, possible defect bands at $2.2 \mathrm{eV}$, as observed in photoconductivity spectroscopy experiments [68], have for a long time shed confusion on the true value of the bandgap. This relatively "small" bandgap has made BFO one of the prominent multiferroics for the study and application of the coupling between light and ferroic order parameters, such 


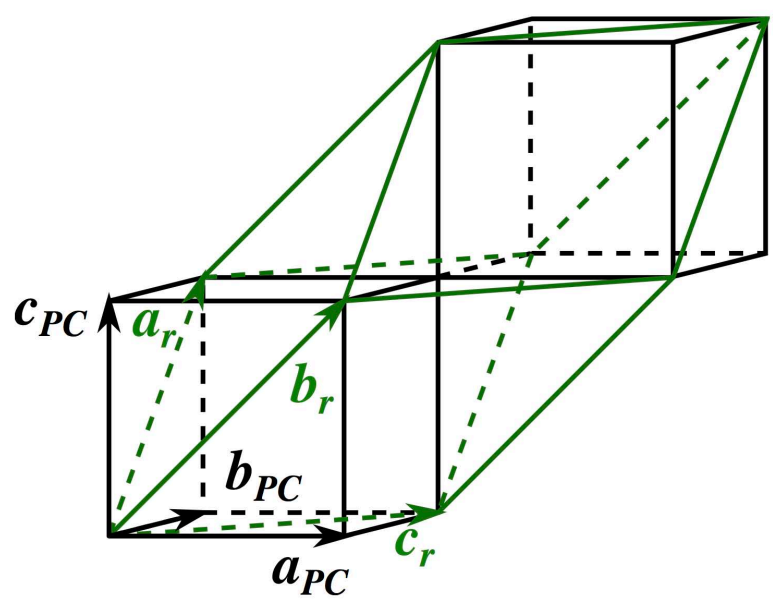

Figure 8: Bismuth ferrite rhombohedral unit cell (green) and pseudocubic 5atom unit cell.

as photovoltaics $[69,70,71]$, photocatalysis $[72,73,21]$ and photostriction $[22$, 15, 26]. Steady photostriction in $\mathrm{BiFeO}_{3}$ was studied in Ref. [74] using the $\triangle \mathrm{SCF}$ method in a similar fashion to lead and barium titanates, except that the LSDA $+U$ functional was used, with $U=3.87 \mathrm{eV}$. The band structure of bismuth ferrite is plotted in Fig. (9). The bottom of the conduction band (CBM) is located at the $Z$ point, while the top of the valence band (VBM) is located in-between $B$ and $Z$ points. Three transitions, depicted by different arrows in Fig. (9), are considered: $Z \longrightarrow Z$ (denoted direct CBM, in green), $V B M \longrightarrow Z$ (in red, denoted indirect VBM-CBM) and a vertical transition from the VBM (in blue, denoted direct VBM).

The results, shown in Fig. (10), show that the pseudocubic directions are shrinking upon photoexcitation. Generally speaking, pseudocubic directions "close" to the polar axis (such as [100], [110] and [111]) tend to shorten. On the other hand, due to the opening of the pseudocubic angle with the increase of photo-excited carriers, the directions "away" from the polar axis (such as [11̄0] or [1111]) lengthen [74].

Interestingly, experiments on single crystals of $\mathrm{BiFeO}_{3}$ under UV light report an elongation of $+2.7 \times 10^{-3} \%$ along the [101] pseudocubic direction [15]. According to our calculations, an expansion is only possible if this $<101>$ direction is actually a $[10 \overline{1}]$ direction (i.e., perpendicular to the spontaneous polarization), in which case such expansion would occur for a concentration of photoexcited electrons of roughly $1.6 \times 10^{19} \mathrm{~cm}^{-3}$. Note that, according to literature reports, concentration of photoexcited carriers ranging from $5 \times 10^{17}$ to $5 \times 10^{19} \mathrm{~cm}^{-3}$ were measured or estimated $[27,75]$.

To answer the question about whether the origin of the calculated photostric- 


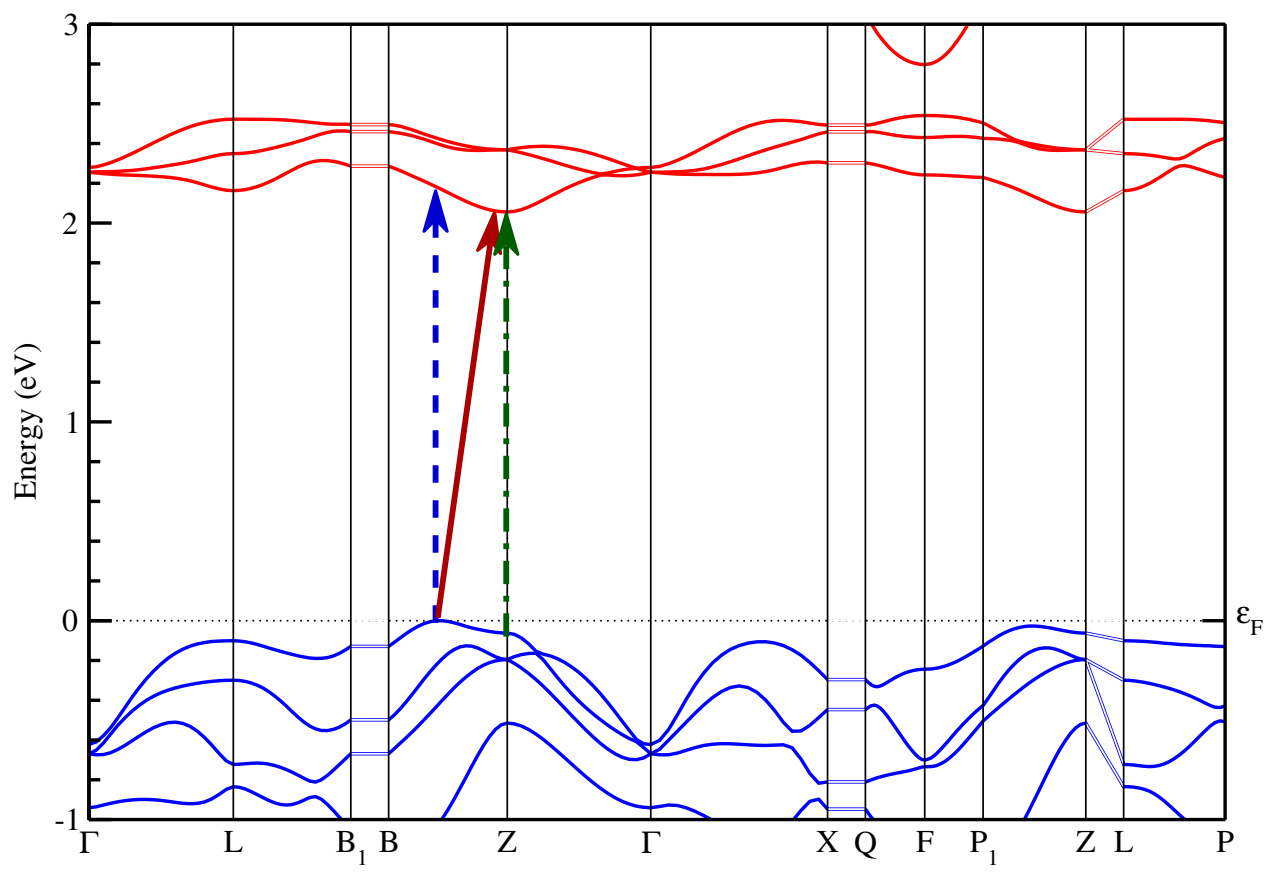

Figure 9: Band structure of $R 3 c$ bismuth ferrite, and the different transitions considered in Ref. [74]. Copyright 2016 American Physical Society.
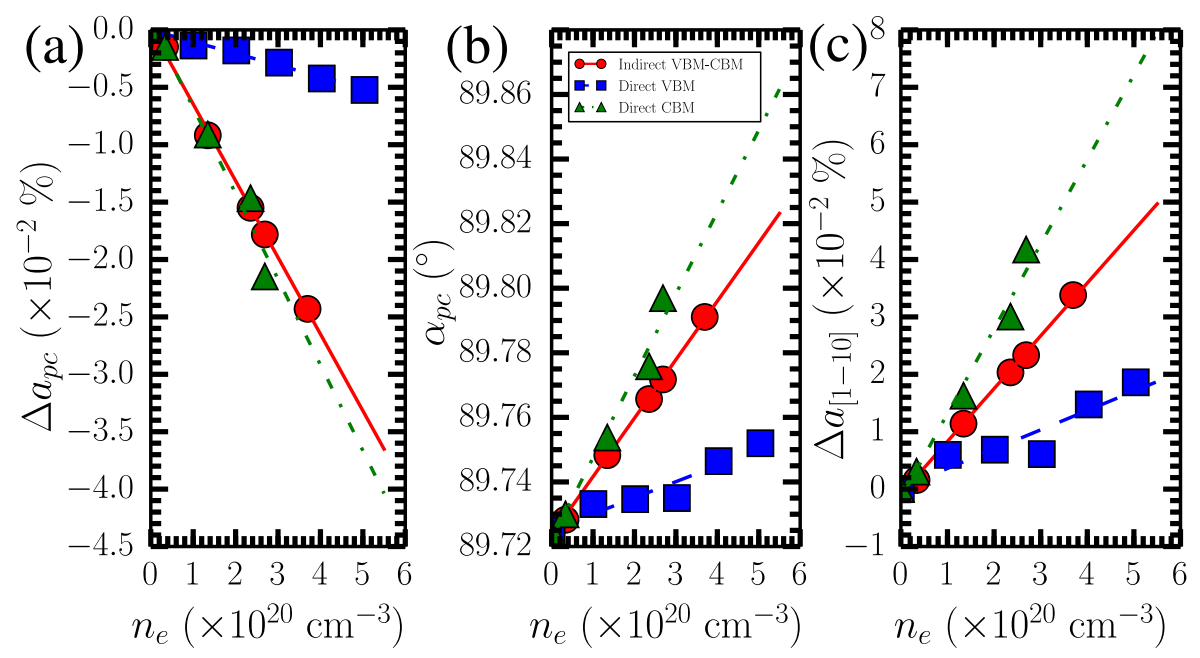

Figure 10: Change of (a) pseudocubic lattice constant, (b) pseudocubic angle and (c) length along the pseudocubic [110] direction with the number of photoexcited carriers in rhombohedral bismuth ferrite. Taken from Ref. [74]. Copyright 2016 American Physical Society. 


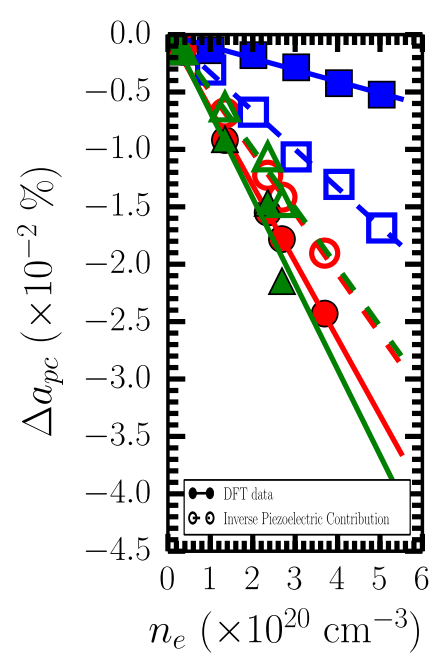

Figure 11: Calculated photo-induced change in pseudocubic lattice constant (filled symbols) and estimated change in lattice constant caused by reduction of the polarization and converse piezoelectric effect (open symbols) in $R 3 c \mathrm{BiFeO}_{3}$. Taken from Ref. [74]. Copyright 2016 American Physical Society.

tive effect was driven by the piezoelectric effect or not, we conducted a similar analysis as in lead and barium titanates. The polarization was shown to diminish with the number of photoexcited carriers $n_{e}$. Meanwhile, the piezoelectric tensor $\boldsymbol{d}$ and the dielectric susceptibility $\chi$, taken from molecular dynamics simulations [76], allowed the estimation of the piezoelectric constant $\boldsymbol{g}$ and therefore of the piezoelectric photo-induced strain (see open symbols in Fig. (11)). It is found that for the direct CBM and indirect VBM-CBM transitions, the piezoelectric model is rather accurate. Performing frozen ion $\triangle \mathrm{SCF}$ calculations indeed proves that the electronic pressure was a minor (cooperating) contribution in those cases [74]. On the other hand, the photo-induced change of lattice constant in the direct VBM transition case is predicted to be stronger in the piezoelectric model than the calculated values, which was found later to be the result of the competition of the piezoelectric mechanism (which in this case favors shrinking) with the potential deformation (which for this transition favors expansion). Overall, the piezoelectric effect still prevails, but its influence is reduced.

\subsection{Conclusions about photostriction in ferroelectrics}

Overall, the use of first-principles tends to confirm the scenario of a converse piezoelectric effect-driven photostriction in ferroelectric materials. This seems to be particularly true along the polar axis, for which the piezoelectric constant is larger. However, in directions perpendicular to the polar axis, the 


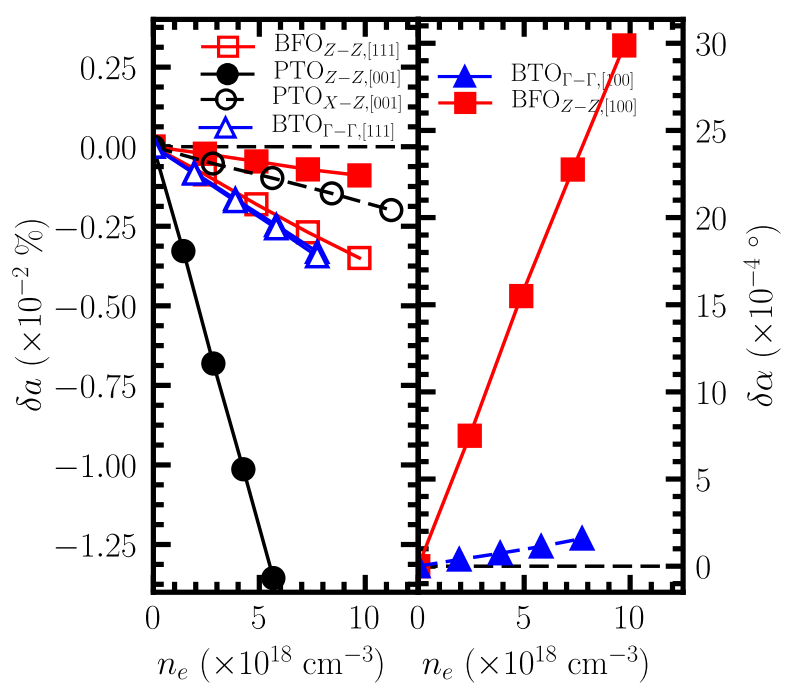

Figure 12: (left panel) Photo-induced change of length in tetragonal PTO, rhombohedral BTO and rhombohedral BFO along several directions, for different transitions; (right panel) Change in pseudocubic angle in rhombohedral BTO versus rhombohedral BFO. Taken from Ref. [42]. Copyright 2017 from the American Physical Society. 
photo-induced potential deformation can become a sizable contribution or competition as demonstrated in the case of lead titanate. Comparing PTO, BTO and BFO in Fig. (12), it appears that lead titanate, at least in the case of the $Z \longrightarrow Z$ transition, has the largest photostriction potential, which results mostly from (i) strong photo-induced change of polarization and (ii) large piezoelectric constant. Note also that two dimensional ferroelectric compounds, such as in the monochalcogenide family $(\mathrm{SnS}, \mathrm{SnSe})$, have also been predicted to be good photostrictive materials using the same methods [77].

\section{Elasto-optic effects in ferroelectrics}

Reference [45] investigated elasto-optic effects in PTO films under epitaxial strain. For that, it first computed the total energy of different phases, as shown in Figure (13a). Note that these calculations also predicted that bulk PTO adopts a tetragonal (T) phase with a space group of $P 4 m m$, and having a lattice constant $a$ equal to $3.87 \AA$ as well as an axial ratio of 1.04 . Such computational results are rather consistent with experimental results of $1.02-1.06$ [78]), and the fact that PTO bulk adopts a $P 4 m m$ state below $760 \mathrm{~K}$ [79]. As shown in Fig. (13a), this tetragonal $\mathrm{T}$ phase is also predicted to be the ground state (i.e., it has the lowest energy) for strains ranging from $0 \%$ to $1 \%$. Its out-of-plane polarization $\left(\mathrm{P}_{z}\right)$ decreases from $0.87 \mathrm{C} / \mathrm{m}^{2}$ to $0.80 \mathrm{C} / \mathrm{m}^{2}$ [see Fig. (13d)], and its axial ratio reduces from 1.04 to 1.02 as the strain increases from 0 to $0.75 \%$ [see Fig. (13b)].

In the strain window from $0.75 \%$ to $1.75 \%$, a monoclinic $C m$ phase first has a very low energy close to that of the $T$ phase and then becomes the ground state [see Fig. (13a)]. Another monoclinic Pm phase exhibits a very similar energy than the $C m$ phase, indicating that both $C m$ and $P m$ can likely be stabilized in PTO films by, e.g., applying epitaxial strain and/or electric field. Interestingly, both $\mathrm{Pm}$ and $\mathrm{Cm}$ phases have been observed for PTO films grown on a $\mathrm{DyScO}_{3}$ substrate (corresponding to a biaxial strain of 1.4\%) [80]. As shown Fig. (13b), the $\mathrm{Cm}$ phase decreases its axial ratio from 1.022 to 0.988 when the epitaxial strain ranges from $0.75 \%$ to $1.75 \%$. Unlike the $T$ phase that has only an out-ofplane component of polarization, the $\mathrm{Cm}$ phase has both out-of-plane and inplane components of polarization. When this strain varies from $0.75 \%$ to $1.75 \%$, the in-plane polarization $\left(\mathrm{P}_{x}\right)$ of $C m$ increases from about zero to $0.66 \mathrm{C} / \mathrm{cm}^{2}$ while the out-of-plane polarization $\mathrm{P}_{z}$ concomitantly decreases from $0.75 \mathrm{C} / \mathrm{cm}^{2}$ to almost zero. In other words and as shown in Fig. (14a), the polarization of the $\mathrm{Cm}$ state continuously rotates from the out-of-plane [001] direction towards the in-plane [110] direction when increasing strain in this intermediate strain range. Regarding the $P m$ monoclinic phase, the axial ratio decreases from values larger than 1 to less than 1 when the strain is enhanced between $0.75 \%$ to $1.75 \%$, and is qualitatively (and even quantitatively) very similar to the axial ratio of the $\mathrm{Cm}$ phase. As shown in Figs. (14c) and (14d), the $\mathrm{P}_{z}$ out-of-plane polarization of $P m$ decreases and the $\mathrm{P}_{x}$ in-plane polarization increases in this tensile strain range. Note that $\mathrm{P}_{y}$ is zero for the $P m$ phase, which differs from the case of the 

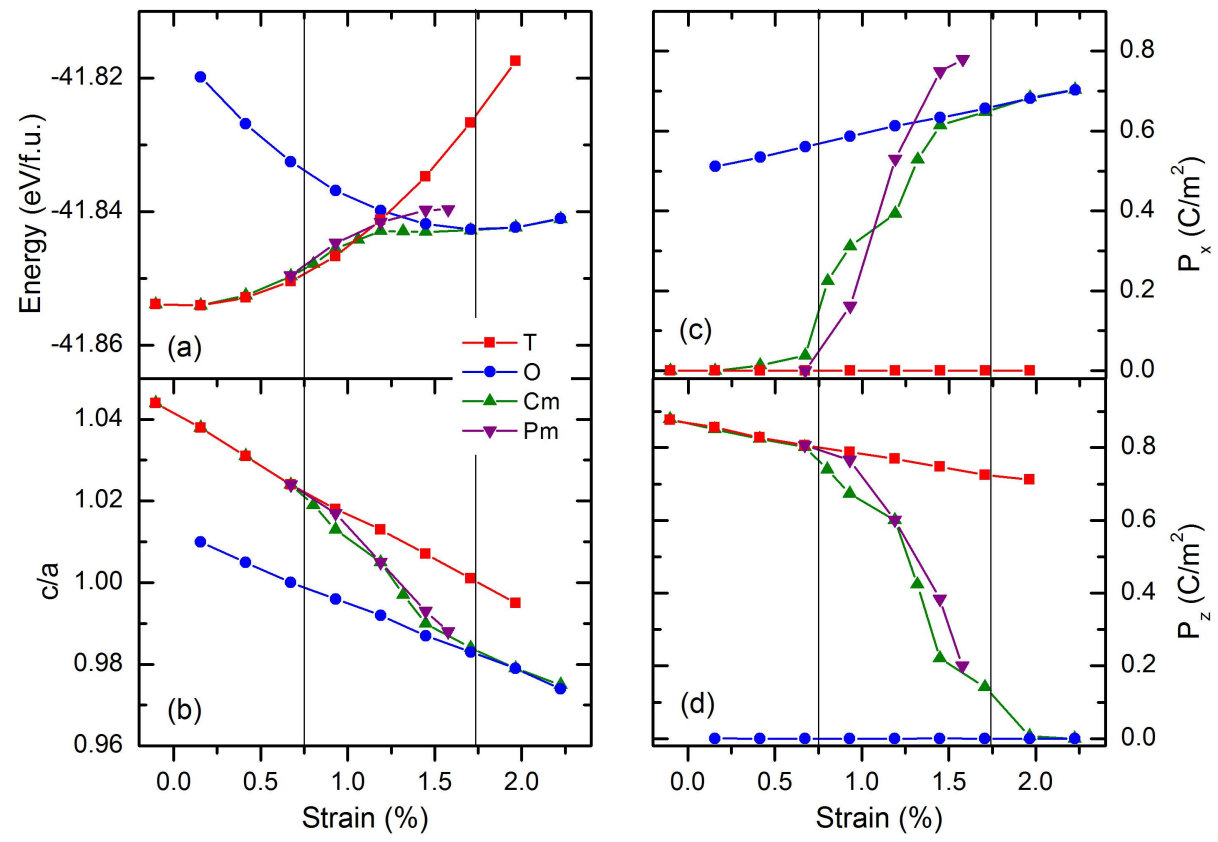

Figure 13: Total energy (a), axial ratio (b), x-component of the polarization, $\mathrm{P}_{x}$ (c), and out-of-plane component of the polarization, $\mathrm{P}_{z}$ (d), of PTO films as a function of tensile strain. Note that $\mathrm{P}_{y}=\mathrm{P}_{x}$ for the $O$ and $C m$ phases, $\mathrm{P}_{y}=0$ for the $T$ and $P m$ phases. Taken from Ref. [45]. Copyright 2016 from the American Physical Society. 
(a)

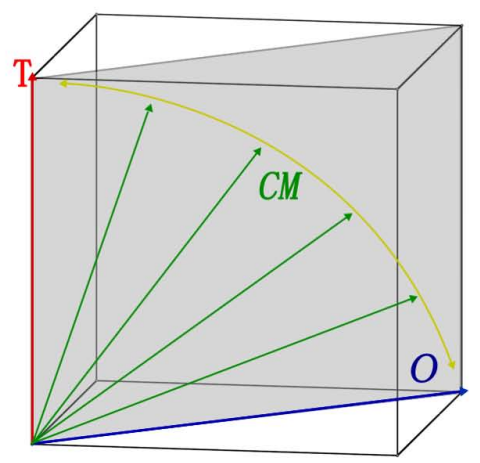

(b)

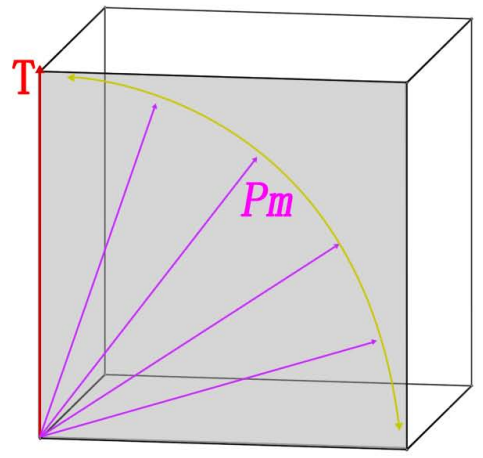

Figure 14: Schematic representations of polarization directions of the $\mathrm{Cm}, \mathrm{Pm}$, $O$, and $T$ phases. Polarization of the $T$ phase is along the [001] direction, of the $O$ phase is along the [110] direction. Polarization of $C m$ is along a [uuv] direction (with $u$ being different with $v$ ), which rotates from the [100] direction to the [110] direction under strain, and polarization of $P m$ is along a $[u 0 v]$ direction (with $u$ being different with $v$ ), which rotates from the [001] direction to the [100] direction under strain.

$C m$ phase for which $\mathrm{P}_{y}=\mathrm{P}_{x}$ is non-null. As shown in Fig. (14b), the polarization of the $P m$ phase rotates from the pseudo-cubic [001] to [100] directions when increasing the tensile strain. These different in-plane polarization can induce different optical properties and elasto-optic effects between the $\mathrm{Cm}$ and $\mathrm{Pm}$ phases, as we will see below.

At the large strain $>1.75 \%$, an orthorhombic $(O)$ phase has the lowest energy and thus becomes the ground state. This $O$ phase has an axial ratio smaller than 1, a large in-plane polarization and a null out-of-plane polarization.

Optical properties and elasto-optic effects were considered in Reference [45] for these different phases of PTO under tensile strain. The predicted refractive index $n$ for an $\hbar \omega$ energy being $0.3 \mathrm{eV}$ below the computed band gap is shown in Fig. (15). $n_{o}$ and $n_{e}$ represent the refractive index for ordinary ray and extraordinary ray, respectively. As shown in Fig. (15a), $n_{o}$ is larger then $n_{e}$ in the $T$ phase, while the reverse situation holds for the $O$ phase. The hierarchy between $n_{o}$ and $n_{e}$ in the $C m$ and $P m$ phases depends on strain. At strain less than about $1.25 \%, n_{o}$ is higher than $n_{e}$, while $n_{o}$ is less than $n_{e}$ for strain larger than $\simeq 1.25 \%$. To further investigate the hierarchy of $n_{o}$ and $n_{e}$, Fig. (15b) shows the refractive index as a function of the axial ratio $c / a$. In first approximation, $n_{o}$ is equal to $n_{e}$ when $c / a$ is 1 , and $n_{o}$ is larger (smaller) than $n_{e}$ when $c / a$ is larger (smaller) than 1. Therefore, the hierarchy between $n_{o}$ and $n_{e}$ is dependent on the $c / a$ axial ratio. Such facts may be useful for discovering materials with large elasto-optic effects.

Furthermore, both $n_{o}$ and $n_{e}$ increase with strain in the $T$ phase and decrease 


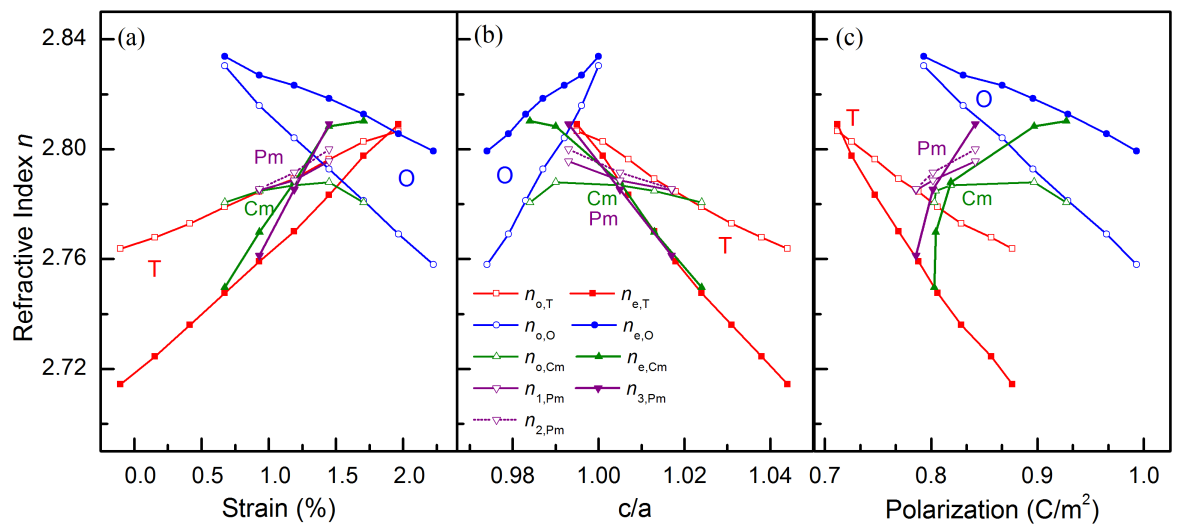

Figure 15: Panel (a): Refractive index $n$ of ordinary (open symbols) and extraordinary rays (filled symbols) for PTO in its $T$ (squares), $O$ (dots) and $C m$ (up triangles) phases as a function of strain. Note that for the monoclinic $\mathrm{Pm}$ phase, the $\mathrm{x}$ - and y-directions are not equivalent by symmetry (due to the polarization direction), and therefore $n_{1}$ and $n_{2}$ differ. In-plane and out-of-plane refractive indices of the $P m$ phase (down triangles) are also shown. Panels (b) and (c): Similar to Panel (a) but as a function of the axial ratio and magnitude of the polarization, respectively. Taken from Ref. [45]. Copyright 2016 American Physical Society.

in the $O$ phase [see Fig. (15a)]. This different behavior is related to polarization. As a matter of fact and as shown in Fig. (15c), both $n_{o}$ and $n_{e}$ decrease when the polarization increases in the $T$ and $O$ phases. As $C m$ and $P m$ phases structurally bridge the $T$ and $O$ phases, the refractive $n_{o}$ and $n_{e}$ indices of these monoclinic state have to continuously evolve between those of the corresponding refractive indices of the $T$ and $O$ phases. $n_{o}$ at strain $0.75 \%$ of the $T$ phase and at the strain $1.5 \%$ in the $O$ phase are very similar, therefore $n_{o}$ in $C m$ and $P m$ phases does not need to change too much to bridge the $T$ and $O$ phases at the intermediate strain of $0.75 \%-1.5 \%$. In contrast, $n_{e}$ at strain $0.75 \%$ of the $T$ phase is much smaller than $n_{e}$ at the strain $1.5 \%$ in the $O$ phase. $n_{e}$ in the $C m$ and $P m$ phases thus have to sharply increase in the intermediate strain to bridge the $n_{e}$ 's of the $T$ and $O$ phases. This sharply changing $n_{e}$ with strain can induce large elasto-optic effects, according to Eq. (16).

As a matter of fact and as revealed in Table 1, the effective elasto-optic coefficient $\left(p_{31}+p_{32}\right) / 2$ for the monoclinic $\mathrm{Cm}$ and $\mathrm{Pm}$ phases indeed has very large values. It is at least two times that of the $T$ phase and three times that of the $O$ state. The large elasto-optic coefficient is related to $n_{e}$ in $C m$ and $n_{3}$ in $P m$, which significantly change with strain. These value appear to be the largest elasto-optic coefficient predicted or reported. For instance, they are about twice as large as the coefficient of lithium niobate, for which $p_{31}=0.18$, and $30-60 \%$ 
Table 1: Elasto-optic coefficients for the four studied phases of epitaxial (001) PTO films under a tensile strain of $1.2 \%$, for an $\hbar \omega$ energy being $0.3 \mathrm{eV}$ smaller than the computed band gap. The number in parentheses corresponds to $\left(p_{21}+\right.$ $\left.p_{22}\right) / 2$ of the $P m$ phase, which, due to symmetry and unlike in the $T, O$ and $C m$ phases, differs from $\left(p_{11}+p_{12}\right) / 2$. Note that $p_{31}=p_{32}$ in the $T, O$ and $C m$ states, therefore making $\left(p_{31}+p_{32}\right) / 2=p_{31}$ in these three phases. Taken from Ref. [45]. Copyright 2016 American Physical Society.

\begin{tabular}{ccccc}
\hline \hline Phase & $T$ & $O$ & $P m$ & $C m$ \\
\hline$\left(p_{11}+p_{12}\right) / 2$ & -0.10 & 0.20 & $-0.09(-0.13)$ & -0.04 \\
$\left(p_{31}+p_{32}\right) / 2$ & -0.22 & 0.10 & -0.43 & -0.35 \\
\hline \hline
\end{tabular}

bigger than that of quartz $\left(p_{31}=0.29\right)[81]$.

In this part of the review, we described the four phases of $\mathrm{PbTiO}_{3}$ under tensile strain, and their refractive indices behavior under strain. The fast increase of refractive index $n_{e}$ of the monoclinic $C m$ and $P m$ phases with epitaxial strain induces very large elasto-optic effects. Further analysis reveals that such rapid change of refractive index is also related to a sharp change of the axial ratio with strain [see Fig. (15b)]. These results may provide a new route to discover large elasto-optic effect materials by searching for low-symmetry phases possessing a rapid evolution of the axial ratio and polarization with strain. Note that this rapid evolution can occur in epitaxial ferroelectrics films $[82,83]$ but also in the morphotropic phase boundaries of some solid solutions[84, 85, 86, 87, 88] when varying composition or hydrostatic pressure. Note also that a recent work demonstrated that large elasto-optic coefficients can occur in epitaxial thin films made of the multiferroic $\mathrm{BiFeO}_{3}$ [89].

\section{Conclusion}

In summary, this review provided details about (i) the methods used to recently predict photostriction and elasto-optic effects in ferroelectrics and multiferroics from first principles; and (ii) their concomitant results and analyses aimed at microscopically understanding such effects. We hope that these methods and analyses can be used as starting points to design novel FE and MFE materials exhibiting giant couplings between light and physical properties. Such interesting general topic is at its infancy stage, and therefore further developments (such as tackling these couplings using the more sophisticated and complex GW [52] and time-dependent DFT [90] schemes as well as exciting related discoveries are likely to occur in a near future.

This work is supported by the ONR Grant N00014-17-1-2818 (Y.Y), the DARPA Grant No. HR0011-15- 2-0038 under the MATRIX program (C.P.) and the U.S. AFOSR Grant No. FA9550-16-1-0065 (B.X. and L.B.). 


\section{References}

[1] M. E. Lines and A. M. Glass. Principles and Applications of Ferroelectrics and Related Materials. Oxford University Press, Oxford, 2001.

[2] G. Catalan and James F. Scott. Physics and Applications of Bismuth Ferrite. Adv. Mater., 21(24):2463-2485, 2009.

[3] Ilya Grinberg, D. Vincent West, Maria Torres, Gaoyang Gou, David M. Stein, Liyan Wu, Guannan Chen, Eric M. Gallo, Andrew R. Akbashev, Peter K. Davies, Jonathan E. Spanier, and Andrew M. Rappe. Perovskite oxides for visible-light-absorbing ferroelectric and photovoltaic materials. Nature, 503(7477):509-512, 2013.

[4] Riad Nechache, C. Harnagea, S. Li, L. Cardenas, W. Huang, Joyprokash Chakrabartty, and Federico Rosei. Bandgap tuning of multiferroic oxide solar cells. Nat. Photonics, 9(1):61-67, 2014.

[5] Jonathan E. Spanier, Vladimir M. Fridkin, Andrew M. Rappe, Andrew R. Akbashev, Alessia Polemi, Yubo Qi, Zongquan Gu, Steve M. Young, Christopher J. Hawley, Dominic Imbrenda, Geoffrey Xiao, Andrew L. Bennett-Jackson, and Craig L. Johnson. Power conversion efficiency exceeding the Shockley-Queisser limit in a ferroelectric insulator. Nat. Photonics, 10(9):611-616, 2016.

[6] Charles Paillard, Xiaofei Bai, Ingrid C. Infante, Maël Guennou, Grégory Geneste, Marin Alexe, Jens Kreisel, and Brahim Dkhil. Photovoltaics with Ferroelectrics: Current Status and Beyond. Adv. Mater., 28(26):5153-5168, 2016.

[7] Pilar Lopez-Varo, Luca Bertoluzzi, Juan Bisquert, Marin Alexe, Mariona Coll, Jinsong Huang, Juan Antonio Jimenez-Tejada, Thomas Kirchartz, Riad Nechache, Federico Rosei, and Yongbo Yuan. Physical aspects of ferroelectric semiconductors for photovoltaic solar energy conversion. Phys. Rep., 653(August):1-40, 2016.

[8] V. M. Fridkin and B. N. Popov. Anomalous photovoltaic effect in ferroelectrics. Soviet Physics Uspekhi, 981, 1978.

[9] V. M. Fridkin, E. P. Efremova, B. H. Karimov, V. A. Kuznezov, I. P. Kuzmina, A. N. Lobachev, V. G. Lazarev, and A. J. Rodin. The experimental investigation of the photovoltaic effect in some crystals without a center of symmetry. Appl. Phys., 25(1):77-80, 1981.

[10] Boris I. Sturman and V. M. Fridkin. The Photovoltaic and Photorefractive Effects in Noncentrosymmetric Materials. Gordon and Breach Science Publishers, 1992.

[11] V. M. Fridkin. Bulk photovoltaic effect in noncentrosymmetric crystals. Crystallogr. Rep., 46(4):654-658, 2001. 
[12] Yongfei Cui, Joe Briscoe, and Steve Dunn. Effect of Ferroelectricity on Solar-Light-Driven Photocatalytic Activity of BaTiO 3 - Influence on the Carrier Separation and Stern Layer Formation. Chem. Mater., 25(21):42154223, 2013.

[13] I. Tatsuzaki, K. Itoh, S. Ueda, and Y. Shindo. Strain along $c$-axis of sbsi caused by illumination in dc electric field. Phys. Rev. Lett., 17(4):198-200, 1966.

[14] Xiao Dingquan, Zhu Jianguo, Zhao Shipin, Wang Xiu, Zhang Wen, Liu Guoqin, and $\mathrm{Xu}$ Guanfeng. Photostriction effect of doped linbo 3 single crystals. Solid State Commun., 79(11):1005-1007, 1991.

[15] B. Kundys, Michel Viret, C. Meny, V. Da Costa, Dorothée Colson, and B. Doudin. Wavelength dependence of photoinduced deformation in bifeo $_{3}$. Phys. Rev. B, 85(9):092301, 2012.

[16] Sheng-Yuan Chu, Zhou Ye, and Kenji Uchino. Impurity doping effect on photostriction in PLZT ceramics. Adv. Perform. Mater., 1(2):129-143, 1994.

[17] Kenji Uchino, Y Miyazawa, and Shoichiro Nomura. High voltage photovoltaic effect in PbTiO3-based ceramics. Jpn. J. Appl. Phys., Part 1, 1671, 1982 .

[18] P. Poosanaas and Kenji Uchino. Photostrictive effect in lanthanummodified lead zirconate titanate ceramics near the morphotropic phase boundary. Materials chemistry and physics, 61:36-41, 1999.

[19] Kenji Uchino. New applications of photostrictive ferroics. Mater. Res. Innov., 1(3):163-168, 1997.

[20] James F. Scott. Ferroelectric Memories, volume 3 of Springer Series in Advanced Microelectronics. Springer Berlin Heidelberg, Berlin, Heidelberg, 2000.

[21] Xiaofei Bai, Jie Wei, Bobo Tian, Yang Liu, Thomas Reiss, Nicolas Guiblin, Pascale Gemeiner, Brahim Dkhil, and Ingrid C. Infante. Size effect on optical and photocatalytic properties in bifeo $_{3}$ nanoparticles. J. Phys. Chem. C, 120(7):3595-3601, 2016.

[22] B. Kundys, Michel Viret, Dorothée Colson, and D O Kundys. Light-induced size changes in BiFeO3 crystals. Nat. Mater., 9(10):803-5, 2010.

[23] V. Iurchuk, D. Schick, J. Bran, D. Colson, A. Forget, D. Halley, A. Koc, M. Reinhardt, C. Kwamen, N. A. Morley, M. Bargheer, Michel Viret, R. Gumeniuk, G. Schmerber, B. Doudin, and B. Kundys. Optical Writing of Magnetic Properties by Remanent Photostriction. Phys. Rev. Lett.s, 117(10):107403, 2016. 
[24] Pascal Ruello, T. Pezeril, S. Avanesyan, Gwenaelle Vaudel, Vitalyi E. Gusev, Ingrid C. Infante, and Brahim Dkhil. Photoexcitation of gigahertz longitudinal and shear acoustic waves in $\mathrm{BiFeO} 3$ multiferroic single crystal. Appl. Phys. Lett., 100(21):212906, 2012.

[25] Dan Daranciang, Matthew J. Highland, Haidan Wen, Steve M. Young, Nathaniel C. Brandt, Harold Y. Hwang, Michael Vattilana, Matthieu Nicoul, Florian Quirin, John Goodfellow, Tingting Qi, Ilya Grinberg, David M. Fritz, Marco Cammarata, Diling Zhu, Henrik T. Lemke, Donald Walko, Eric M. Dufresne, Yuelin Li, Jörgen Larsson, David a. Reis, Klaus Sokolowski-Tinten, Keith a. Nelson, Andrew M. Rappe, Paul H. Fuoss, G. Brian Stephenson, and Aaron M. Lindenberg. Ultrafast Photovoltaic Response in Ferroelectric Nanolayers. Phys. Rev. Lett., 108(8):087601, 2012.

[26] Haidan Wen, Pice Chen, Margaret P Cosgriff, Donald Walko, June Lee, Carolina Adamo, Richard D Schaller, Jon Ihlefeld, Eric M. Dufresne, Darrell G. Schlom, Paul Evans, John Freeland, and Yuelin Li. Electronic origin of ultrafast photoinduced strain in bifeo $_{3}$. Phys. Rev. Lett., 110(3):037601, 2013.

[27] Mariusz Lejman, Gwenaelle Vaudel, Ingrid C. Infante, Pascale Gemeiner, Vitalyi E. Gusev, Brahim Dkhil, and Pascal Ruello. Giant ultrafast photoinduced shear strain in ferroelectric BiFeO3. Nat. Commun., 5:4301, 2014.

[28] Daniel Schick, Marc Herzog, Haidan Wen, Pice Chen, Carolina Adamo, Peter Gaal, Darrell G. Schlom, Paul G. Evans, Yuelin Li, and Matias Bargheer. Localized excited charge carriers generate ultrafast inhomogeneous strain in the multiferroic bifeo $_{3}$. Phys. Rev. Lett., 112(9):097602, 2014.

[29] Haidan Wen, Michel Sassi, Zhenlin Luo, Carolina Adamo, Darrell G. Schlom, Kevin M. Rosso, and Xiaoyi Zhang. Capturing ultrafast photoinduced local structural distortions of bifeo $_{3}$. Sci. Rep., 5(October):15098, 2015 .

[30] Yuelin Li, Carolina Adamo, Pice Chen, Paul G Evans, Serge M Nakhmanson, William Parker, Clare E Rowland, Richard D Schaller, Darrell G. Schlom, Donald a Walko, Haidan Wen, and Qingteng Zhang. Giant optical enhancement of strain gradient in ferroelectric bifeo 3 thin films and its physical origin. Sci. Rep., 5(1):16650, 2015.

[31] Mariusz Lejman, Gwenaelle Vaudel, Ingrid C. Infante, Ievgeniia Chaban, Thomas Pezeril, Mathieu Edely, Guillaume F. Nataf, Mael Guennou, Jens Kreisel, Vitalyi E. Gusev, Brahim Dkhil, and Pascal Ruello. Ultrafast acousto-optic mode conversion in optically birefringent ferroelectrics. Nat. Commun., 7:12345, 2016.

[32] B. Kundys. Photostrictive materials. Appl. Phys. Rev., 2(1):011301, 2015. 
[33] C. Thomsen, H. T. Grahn, H. J. Maris, and J. Tauc. Surface generation and detection of phonons by picosecond light pulses. Phys. Rev. B, 34(6):4129$4138,1986$.

[34] Pascal Ruello and Vitalyi E. Gusev. Physical mechanisms of coherent acoustic phonons generation by ultrafast laser action. Ultrasonics, 56(July 2016):21-35, 2015.

[35] J. Bardeen and W. Shockley. Deformation Potentials and Mobilities in Non-Polar Crystals. Phys. Rev., 80(1):72-80, 1950.

[36] Conyers Herring and Erich Vogt. Transport and Deformation-Potential Theory for Many-Valley Semiconductors with Anisotropic Scattering. Phys. Rev., 101(3):944-961, 1956.

[37] B. Noheda, D. E. Cox, G. Shirane, J. A. Gonzalo, L. E. Cross, and S-E. Park. A monoclinic ferroelectric phase in the $\mathrm{pb}\left(\mathrm{zr}_{1-x} \mathrm{ti}_{x}\right) \mathrm{o}_{3}$ solid solution. Appl. Phys. Lett., 74(14):2059-2061, 1999.

[38] Laurent Bellaiche, Alberto García, and David Vanderbilt. FiniteTemperature Properties of $\mathrm{Pb}\left(\mathrm{Zr}_{-}\{1-\mathrm{x}\} \$ \mathrm{Ti} \$ \mathrm{x} \$\right) \mathrm{O} \$ 3 \$$ Alloys from First Principles. Phys. Rev. Lett., 84(23):5427-5430, 2000.

[39] B. Noheda, D. E. Cox, G. Shirane, J. Gao, and Zuo-Guang Ye. Phase diagram of the ferroelectric relaxor $(1-x) \mathrm{pbmg}_{1 / 3} \mathrm{nb}_{2 / 3} \mathrm{O}_{3}-x \mathrm{pbtio}_{3}$. Phys. Rev. B, 66(5):054104, 2002.

[40] V A Shuvaeva, A M Glazer, and D Zekria. The macroscopic symmetry of $\mathrm{pb}\left(\mathrm{mg}_{1 / 3} \mathrm{nb}_{2 / 3}\right)_{1-x} \mathrm{ti}_{x} \mathrm{O}_{3}$ in the morphotropic phase boundary region $(x=$ 0.25 - 0.5). J. Phys. Condens. Matter, 17(37):5709-5723, 2005.

[41] Xiangyong Zhao, Bijun Fang, Hu Cao, Yiping Guo, and Haosu Luo. Dielectric and piezoelectric performance of PMN-PT single crystals with compositions around the MPB: influence of composition, poling field and crystal orientation. Mater. Sci. Eng., B, 96(3):254-262, 2002.

[42] Charles Paillard, Sergey Prosandeev, and Laurent Bellaiche. Ab initio approach to photostriction in classical ferroelectric materials. Phys. Rev. B, 96(4):045205, 2017.

[43] R E Pasynkov. On some problems of the phenomenological theory of ferroelectric-semiconductors. Ferroelectrics, 6(1):19-27, 1973.

[44] IC Chang. I. acoustooptic devices and applications. IEEE Trans. Sonics Ultrason, 23(1):2-21, 1976.

[45] Lan Chen, Yurong Yang, Zhigang Gui, D. Sando, M. Bibes, X. K. Meng, and L. Bellaiche. Large elasto-optic effect in epitaxial $\mathrm{pbtio}_{3}$ films. Phys. Rev. Lett., 115:267602, 2015. 
[46] P. Hohenberg and W. Kohn. Inhomogeneous Electron Gas. Phys. Rev., 136(3B):B864-B871, 1964.

[47] W. Kohn and L. J. Sham. Self-Consistent Equations Including Exchange and Correlation Effects. Phys. Rev., 140(4A):A1133-A1138, 1965.

[48] Richard M. Martin. Electronic Structure: Basic Theory and Practical Methods. Cambridge University Press, Cambridge, England, 2004.

[49] Jeppe Gavnholt, Thomas Olsen, Mads Engelund, and Jakob Schiøtz. $\delta$ self-consistent field method to obtain potential energy surfaces of excited molecules on surfaces. Phys. Rev. B, 78(7):075441, 2008.

[50] Kohji Nakamura, Yukie Kitaoka, Toru Akiyama, Tomonori Ito, M. Weinert, and A. J. Freeman. Constraint density functional calculations for multiplets in a ligand-field applied to Fe-phthalocyanine. Phys. Rev. B, 85(23):235129, 2012 .

[51] John P. Perdew. Density functional theory and the band gap problem. Int. J. Quantum Chem., 30(3):451-451, 1986.

[52] Lars Hedin. New Method for Calculating the One-Particle Green's Function with Application to the Electron-Gas Problem. Phys. Rev., 139(3A):A796A823, 1965.

[53] C. A. Ullrich. Time-Dependent Density-Functional Theory, Concepts and Applications. Oxford University Press, 2012.

[54] Jack Deslippe, Georgy Samsonidze, David A. Strubbe, Manish Jain, Marvin L. Cohen, and Steven G. Louie. BerkeleyGW: A massively parallel computer package for the calculation of the quasiparticle and optical properties of materials and nanostructures. Comput. Phys. Commun., 183(6):12691289, 2012.

[55] M. Gajdoš, K. Hummer, G. Kresse, J. Furthmüller, and F. Bechstedt. Linear optical properties in the projector-augmented wave methodology. Phys. Rev. B, 73:045112, 2006.

[56] Huaxiang Fu and Ronald E. Cohen. Polarization rotation mechanism for ultrahigh electromechanical response in single-crystal piezoelectrics. Nature, 403(6767):281-283, 2000. 10.1038/35002022.

[57] G H Kwei, a C Lawson, S J L Billinge, and S W Cheong. Structures of the ferroelectric phases of barium titanate. J. Phys. Chem., 97(10):2368-2377, 1993.

[58] A. M. Glazer. The classification of tilted octahedra in perovskites. Acta Crystallogr. Sect. B Struct. Crystallogr. Cryst. Chem., 28(11):3384- $\overline{3392,}$ 1972. 
[59] Christian Michel, Jean-Michel Moreau, Gary D. Achenbach, Robert Gerson, and W. J. James. The atomic structure of BiFeO3. Solid State Commun., 7(9):701-704, 1969.

[60] Jean-Michel Moreau, Christian Michel, R. Gerson, and W. J. James. Ferroelectric $\mathrm{BiFeO} 3 \mathrm{X}$-ray and neutron diffraction study. J. Phys. Chem. Solids, 32(6):1315-1320, 1971.

[61] F. Kubel and Hans Schmid. Structure of a ferroelectric and ferroelastic monodomain crystal of the perovskite BiFeO3. Acta Crystallogr. Sect. B Struct. Sci., 46(6):698-702, 1990.

[62] Delphine Lebeugle, Dorothée Colson, Anne Forget, Michel Viret, P. Bonville, J. F. Marucco, and S. Fusil. Room-temperature coexistence of large electric polarization and magnetic order in bifeo 3 single crystals. Phys. Rev. B, 76(2):024116, 2007.

[63] Delphine Lebeugle, Dorothée Colson, A. Forget, and Michel Viret. Very large spontaneous electric polarization in bifeo $_{3}$ single crystals at room temperature and its evolution under cycling fields. Appl. Phys. Lett., 91(2):022907, 2007.

[64] C. Blaauw and F. van der Woude. Magnetic and structural properties of BiFeO3. J. Phys. C Solid State Phys., 6:1422, 1973.

[65] Izabela Sosnowska, T P Neumaier, and E Steichele. Spiral magnetic ordering in bismuth ferrite. J. Phys. C Solid State Phys., 15(23):4835-4846, 1982 .

[66] Delphine Lebeugle, Dorothée Colson, A. Forget, Michel Viret, a. M. Bataille, and A. Gukasov. Electric-Field-Induced Spin Flop in BiFeO_\{ $\{3\}$ Single Crystals at Room Temperature. Phys. Rev. Lett., 100(22):227602, 2008.

[67] P. Rovillain, M. Cazayous, Y. Gallais, A. Sacuto, R. P. S. M. Lobo, D. Lebeugle, and D. Colson. Polar phonons and spin excitations coupling in multiferroic bifeo 3 crystals. Phys. Rev. B, 79(18):180411, 2009.

[68] Mingmin Yang, Akash Bhatnagar, and Marin Alexe. Electronic Origin and Tailoring of Photovoltaic Effect in BiFeO 3 Single Crystals. Adv. Electron. Mater., 1(11):1500139, 2015.

[69] T. Choi, S. Lee, Y. J. Choi, V. Kiryukhin, and Sang-Wook Cheong. Switchable ferroelectric diode and photovoltaic effect in bifeo 3 . Science, 324(5923):63-6, 2009.

[70] S.-Y. Yang, L. W. Martin, S. J. Byrnes, T. E. Conry, S. R. Basu, D. Paran, L. Reichertz, Jon Ihlefeld, Carolina Adamo, Alexander Melville, Ying Hao Chu, C.-H. Yang, J. L. Musfeldt, Darrell G. Schlom, J. W. Ager, and Ramamoorthy Ramesh. Photovoltaic effects in bifeo 3 . Appl. Phys. Lett., 95(6):062909, 2009. 
[71] Marin Alexe and Dietrich Hesse. Tip-enhanced photovoltaic effects in bismuth ferrite. Nat. Commun., 2:256, 2011.

[72] Jian He, Renqing Guo, Liang Fang, Wen Dong, Fengang Zheng, and Mingrong Shen. Characterization and visible light photocatalytic mechanism of size-controlled bifeo 3 nanoparticles. Mater. Res. Bull., 48(9):3017-3024, 2013.

[73] Tong Gao, Zhi Chen, Yuxiang Zhu, Feng Niu, Qiaoli Huang, Laishun Qin, Xingguo Sun, and Yuexiang Huang. Synthesis of bifeo $_{3}$ nanoparticles for the visible-light induced photocatalytic property. Mater. Res. Bull., 59:6$12,2014$.

[74] Charles Paillard, Bin Xu, Brahim Dkhil, Grégory Geneste, and Laurent Bellaiche. Photostriction in Ferroelectrics from Density Functional Theory. Phys. Rev. Lett.s, 116(24):247401, 2016.

[75] K. Prashanthi, P. Dhandharia, N. Miriyala, R. Gaikwad, D. Barlage, and T. Thundat. Enhanced photo-collection in single bifeo $_{3}$ nanowire due to carrier separation from radial surface field. Nano Energy, 13(FEBRUARY):240-248, 2015.

[76] M. Graf, M. Sepliarsky, R. Machado, and M.G. Stachiotti. Dielectric and piezoelectric properties of bifeo $_{3}$ from molecular dynamics simulations. Solid State Commun., 218(JUNE):10-13, 2015.

[77] Raad Haleoot, Charles Paillard, Thaneshwor P. Kaloni, Mehrshad Mehboudi, Bin Xu, Laurent Bellaiche, and Salvador Barraza-Lopez. Photostrictive Two-Dimensional Materials in the Monochalcogenide Family. Phys. Rev. Lett., 118(22):227401, 2017.

[78] Gen Shirane, Sadao Hoshino, and Kazuo Suzuki. X-ray study of the phase transition in lead titanate. Phys. Rev., 80:1105-1106, 1950.

[79] Ronald E Cohen. Origin of ferroelectricity in perovskite oxides. Nature, 358(6382):136-138, 1992.

[80] G. Catalan, A. Janssens, G. Rispens, S. Csiszar, O. Seeck, G. Rijnders, D. H. A. Blank, and B. Noheda. Polar domains in lead titanate films under tensile strain. Phys. Rev. Lett., 96:127602, 2006.

[81] Michael Bass, Casimer DeCusatis, and Jay Enoch. Handbook of optics, volume 2. McGraw-Hill New York, 2001.

[82] Gustau Catalan, A Lubk, AHG Vlooswijk, E Snoeck, C Magen, A Janssens, G Rispens, G Rijnders, DHA Blank, and B Noheda. Flexoelectric rotation of polarization in ferroelectric thin films. Nat. Mater., 10(12):963, 2011.

[83] RJ Zeches, MD Rossell, JX Zhang, AJ Hatt, Q He, C-H Yang, A Kumar, CH Wang, A Melville, C Adamo, et al. A strain-driven morphotropic phase boundary in bifeo3. Science, 326(5955):977-980, 2009. 
[84] Y. M. Jin, Y. U. Wang, A. G. Khachaturyan, J. F. Li, and D. Viehland. Conformal miniaturization of domains with low domain-wall energy: Monoclinic ferroelectric states near the morphotropic phase boundaries. Phys. Rev. Lett., 91:197601, 2003.

[85] Tom TA Lummen, Yijia Gu, Jianjun Wang, Shiming Lei, Fei Xue, Amit Kumar, Andrew T Barnes, Eftihia Barnes, Sava Denev, Alex Belianinov, et al. Thermotropic phase boundaries in classic ferroelectrics. Nat. Commun., 5:3172, 2014.

[86] Muhtar Ahart, Maddury Somayazulu, RE Cohen, P Ganesh, Przemyslaw Dera, Ho-kwang Mao, Russell J Hemley, Yang Ren, Peter Liermann, and Zhigang $\mathrm{Wu}$. Origin of morphotropic phase boundaries in ferroelectrics. Nature, 451(7178):545, 2008.

[87] J. Rouquette, J. Haines, V. Bornand, M. Pintard, Ph. Papet, W. G. Marshall, and S. Hull. Pressure-induced rotation of spontaneous polarization in monoclinic and triclinic pbzr $_{0.52} \mathrm{ti}_{0.48} \mathrm{O}_{3}$. Phys. Rev. B, 71:024112, 2005.

[88] Zhigang Wu and Ronald E. Cohen. Pressure-induced anomalous phase transitions and colossal enhancement of piezoelectricity in pbtio $_{3}$. Phys. Rev. Lett., 95:037601, 2005.

[89] D Sando, Yurong Yang, E Bousquet, C Carrétéro, V Garcia, S Fusil, D Dolfi, A Barthélémy, Ph Ghosez, L Bellaiche, et al. Large elasto-optic effect and reversible electrochromism in multiferroic bifeo3. Nat. Commun., 7:10718, 2016.

[90] Carsten A Ullrich. Time-Dependent Density-Functional Theory: Concepts and Applications. Oxford University Press, Oxford, 2011. 